ARTICLE

\title{
R-spondins are BMP receptor antagonists in Xenopus early embryonic development
}

\author{
Hyeyoon Lee ${ }^{1}$, Carina Seidl (10 ${ }^{1}$, Rui Sun ${ }^{1}$, Andrey Glinka ${ }^{1} \&$ Christof Niehrs ${ }^{1,2 \times}$
}

BMP signaling plays key roles in development, stem cells, adult tissue homeostasis, and disease. How BMP receptors are extracellularly modulated and in which physiological context, is therefore of prime importance. R-spondins (RSPOs) are a small family of secreted proteins that co-activate WNT signaling and function as potent stem cell effectors and oncogenes. Evidence is mounting that RSPOs act WNT-independently but how and in which physiological processes remains enigmatic. Here we show that RSPO2 and RSPO3 also act as BMP antagonists. RSPO2 is a high affinity ligand for the type I BMP receptor BMPR1A/ALK3, and it engages ZNRF3 to trigger internalization and degradation of BMPR1A. In early Xenopus embryos, Rspo2 is a negative feedback inhibitor in the BMP4 synexpression group and regulates dorsoventral axis formation. We conclude that R-spondins are bifunctional ligands, which activate WNT- and inhibit BMP signaling via ZNRF3, with implications for development and cancer.

\footnotetext{
${ }^{1}$ Division of Molecular Embryology, DKFZ-ZMBH Alliance, Deutsches Krebsforschungszentrum (DKFZ), 69120 Heidelberg, Germany. ${ }^{2}$ Institute of Molecular Biology (IMB), 55128 Mainz, Germany. ${ }^{凶}$ email: niehrs@dkfz-heidelberg.de
} 
B one Morphogenetic Proteins (BMPs) are a subfamily of TGF $\beta$ growth factors that exert a plethora of crucial functions in embryonic development, adult tissue homeostasis, as well as regeneration, and they underlie human pathology such as skeletal disorders, cancer, and fibrosis in multiple organs ${ }^{1-5}$. Due to their accessibility, extracellular components of the BMP pathway are of particular interest as therapeutic targets ${ }^{6}$ and mechanistic understanding of receptor modulation should improve the ability to manipulate BMP-dependent processes.

BMPs signal through a tetrameric receptor kinase complex composed of type I (BMPR1A/ALK3, BMPR1B/ALK6, ACVR1/ ALK2, or ACVRL1/ALK1) and type II receptors (BMPR2, ACVR2A, ACVR2B) ${ }^{7}$. Ligands and receptors combine in a combinatorial fashion ${ }^{8}$ and phosphorylate SMAD1, 5, and 8, which enter the nucleus with SMAD4 to regulate target gene expression ${ }^{9,10}$. There exists a multitude of extracellular modulators of TGF $\beta$ signaling, either soluble or membrane-associated proteins that control ligand availability, processing, ligand-receptor interaction, and receptor activation ${ }^{11}$. However, only two BMP receptor antagonists are known, which directly bind and inhibit receptor function, the TGF $\beta$-family proteins BMP3 and Inhibin ${ }^{12,13}$.

R-spondins (RSPO1-4) are a family of four secreted $\sim 30 \mathrm{kDa}$ proteins implicated in development and cancer ${ }^{14-20}$. RSPOs are a key ingredient to maintain organoid cultures where they stimulate stem cell growth ${ }^{21,22}$. They amplify WNT signaling by preventing Frizzled/LRP5/6 receptor ubiquitination and degradation via transmembrane E3 ubiquitin ligases ring finger 43 (RNF43) and zinc and ring finger 3 (ZNRF3), thereby sensitizing cells to WNT ligands ${ }^{14,23-25}$. RSPOs bind to ZNRF3/RNF43 and to the stem cell marker Leucine-rich repeat containing $G$ protein-coupled receptor 5 (LGR5), and two related proteins, LGR4 and LGR6, leading to the internalization of the RSPO-LGR-ZNRF3/RNF43 complex and lysosomal degradation $14,17,26$. RSPOs harbor two furin-like repeats (FU1, FU2) domains that bind to ZNRF3/RNF43 and LGRs, respectively ${ }^{27}$. In addition, they contain a thrombospondin 1 (TSP1) domain, which possess about $40 \%$ overall sequence homology $\mathrm{y}^{24,28}$. The TSP1 domain is not essential for WNT/ LRP6 signaling but it binds to HSPGs (Heparan Sulfate Proteoglycans) and thereby promotes WNT5A/PCP (planar cell polarity) signaling ${ }^{24,29}$.

Unexpectedly, recent studies showed that RSPO2 and RSPO3 can potentiate WNT signaling in the absence of all three LGRs in vitro and in vivo ${ }^{27,30}$. Moreover, WNT and RSPO ligands are functionally non-equivalent since e.g., WNT ligand overexpression cannot induce crypt expansion in contrast to RSPO2 or RSPO 31 and RSPO2 and WNT1 have distinct effects on mammary epithelial cell growth ${ }^{32}$ and cochlea development ${ }^{33}$. Hence, these inconsistencies in our current understanding raise the questions: do RSPOs possess WNT-independent functions? Do they engage other receptors? If so, in which physiological processes is this relevant?

Here we show that RSPO2 and RSPO3 are high affinity ligands for the BMP receptor BMPR1A/ALK3. RSPO2 forms a ternary complex between BMPR1A and the E3 ligase ZNRF3, which triggers endocytosis and degradation of the BMP receptor. We show that Rspo2 antagonizes BMP signaling during embryonic axis formation in Xenopus. By gain-of-function and loss-of-function experiments rspo2 cooperates with Spemann organizer effectors to regulate the BMP morphogen gradient, which controls dorsoventral axis formation. Our study reveals R-spondins as BMP receptor antagonists in development, inviting re-interpretation of the mode of action of R-spondins and ZNRF3 in stem cell and cancer biology.

\section{Results}

RSPO2 and RSPO3 antagonize BMP4 signaling independently of WNT. In considering possible WNT-independent functions of
RSPOs, we revisited our early observation that rspo2 overexpression affected BMP signaling in Xenopus embryos ${ }^{20}$. We tested if RSPO2 could suppress BMP signaling in human cells. To this end, we utilized human hepatocellular carcinoma (HEPG2) cells, which express very low levels of RSPOs (Supplementary Fig. 1a). Intriguingly, treatment with RSPO2 and RSPO3 but not RSPO1 and RSPO4 decreased BMP4 signaling, while all RSPOs showed similar ability to amplify WNT signaling (Fig. 1a, Supplementary Fig. 1b). Importantly, inhibition of BMP signaling by RSPO 2 and RSPO3 was independent of $\mathrm{WNT} / \beta$-catenin signaling, since it remained unaffected by siRNA knockdown of $\beta$ catenin (Fig. 1b, Supplementary Fig. 1c, d). RSPO2 and RSPO3, but not RSPO1 and RSPO4 treatment decreased phosphorylation of Smad1, which is a hallmark of BMP signaling activation (Fig. 1c, d, Supplementary Fig. 1e, f). Focusing on RSPO2, we confirmed that RSPO2 overexpression decreased Smad1 phosphorylation and treatment with RSPO2 protein decreased BMP target ID1 expression (Supplementary Fig. 1g, Fig. 1e). Inhibition of BMP signaling by RSPO2 was unaffected by siRNA knockdown of $L G R 4 / 5, L R P 5 / 6, D V L 1 / 2 / 3$, and ROR1/2 (Fig. 1f, g, Supplementary Fig. $1 \mathrm{~h}-\mathrm{j}$ ), suggesting independence of WNT/LRP and WNT/PCP signaling. Moreover, different from RSPO2, treatment with WNT3A, WNT3A surrogate ${ }^{34}$, or the WNT antagonist DKK1 had no effect on BMP signaling (Fig. 1h, Supplementary Fig. 1k, 1), corroborating WNT-independent RSPO2 function.

To delineate the domains required for BMP inhibition, we analyzed deletion mutants of RSPO2 and found both the TSP1domains and FU-domains to be important for signaling inhibition (Fig. 1i, j) ${ }^{24}$. We next investigated RSPO2 deficiency in H1581 cells, a human large cell lung carcinoma cell line that expresses high levels of RSPO2 (Supplementary Fig. 1a). Knockdown of RSPO2 but not LRP5/6 sensitized H1581 cells to BMP stimulation (Fig. 1k, 1, Supplementary Fig. 1m, n). We conclude that RSPO2 and RSPO3 antagonize BMP signaling independently of WNT signaling.

Rspo2 antagonizes BMP signaling during Xenopus embryonic axis development. To analyze if Rspo2 inhibits BMP signaling in vivo, we turned to early Xenopus development. In the early amphibian embryo, the Spemann organizer is a small evolutionary conserved signaling center, which plays an eminent role in regulating embryonic axis formation and neural induction. One essential molecular mechanism underlying Spemann organizer function resides in its secretion of BMP antagonists, which create a BMP morphogen gradient that patterns the embryo ${ }^{35-37}$. Since rspo 2 is expressed and functions in WNT-mediated myogenesis of early Xenopus embryos ${ }^{20}$, we analyzed if it may have an additional role as BMP antagonist in axial patterning.

bmp4 overexpression ventralizes Xenopus embryos, resulting in small heads and enlarged ventral structures ${ }^{38}$. Injection of wildtype $r s p o 2$ mRNA, but neither its $\Delta$ FU1/2 nor $\Delta$ TSP1 deletion mutants rescued these $b m p 4$-induced malformations (Fig. 2a, b). This domain requirement is different from that for WNT signaling activation, where only FU1 and FU2 but not the TSP1 domain are essential ${ }^{20}$. Conversely, injection of a previously characterized rspo2 antisense Morpholino (Mo) ${ }^{20}$ increased endogenous BMP signaling, and this was unaffected by $\operatorname{lrp} 6 \mathrm{Mo}$ (Fig. 2c) ${ }^{39}$. Strikingly, coinjection of bmp4 Mo and rspo2 Mo neutralized each other in BMP signaling reporter assay (Fig. 2d), BMP target gene expression (vent1, sizzled) (Fig. 2e, f), as well as defects in dorsoventral axis development (Supplementary Fig. 2a, b). Typically, overexpression of common BMP antagonists such as noggin or chordin that sequester BMP ligands, leads to strongly dosalized Xenopus embryos, with enlarged heads and cement 

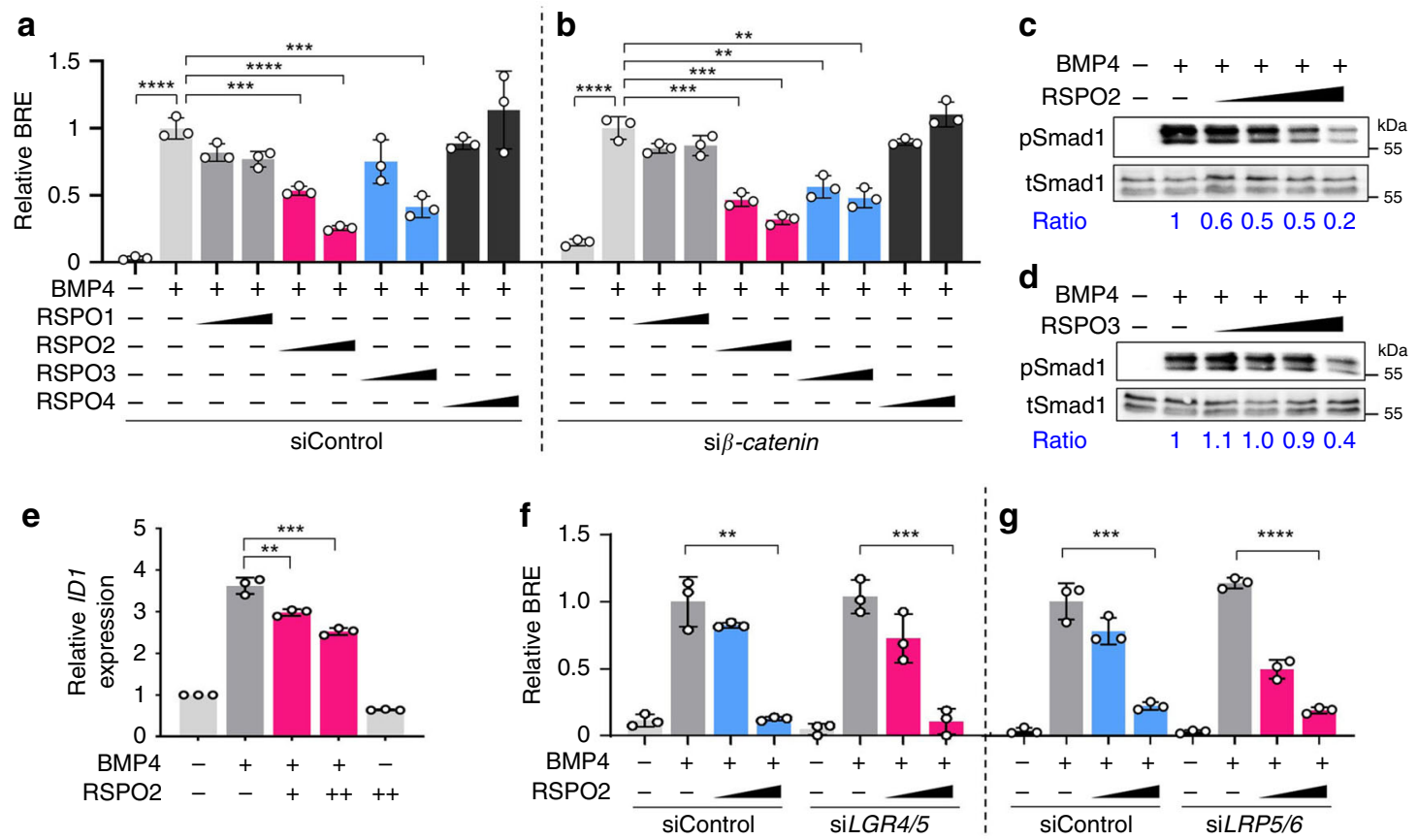

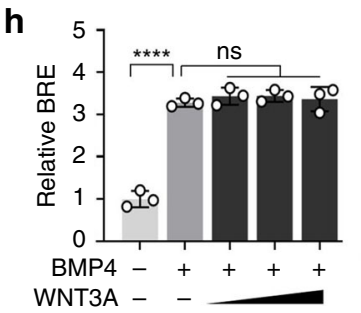

i

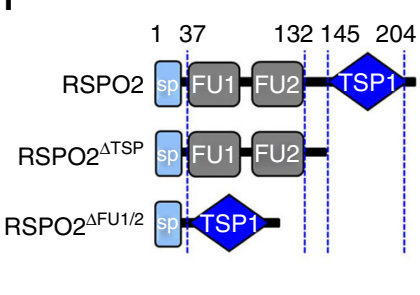

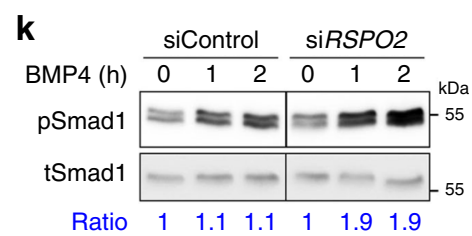
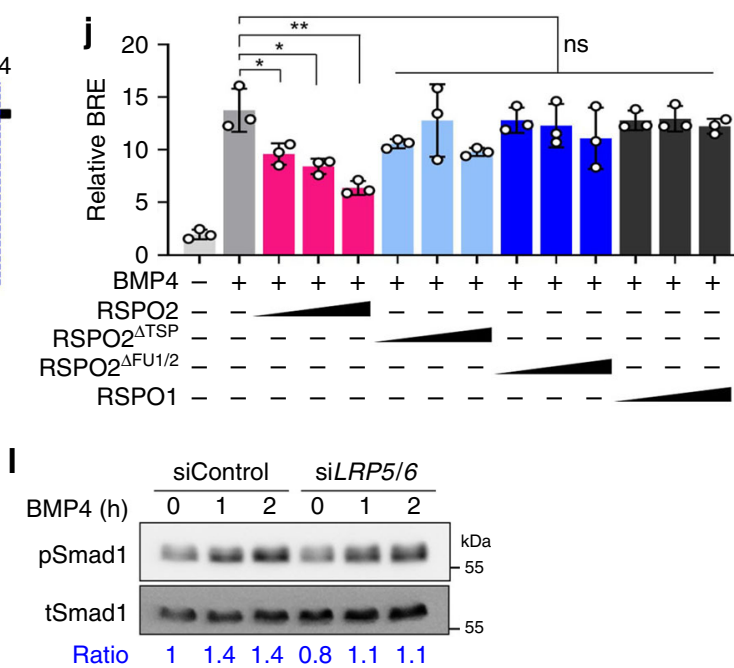

Fig. 1 RSPO2 and RSPO3 antagonize BMP4 signaling WNT independently. a, b BRE reporter assay in HEPG2 cells upon siControl (a) or si $\beta$-catenin (b) transfection, with or without overnight BMP4 and RSPO1-4 treatment as indicated. $n=3$ biologically independent samples. c, $\mathbf{d}$ Western blot analyses of phosphorylated Smad1 (pSmad1) and total Smad1 (tSmad1) in HEPG2 cells stimulated by BMP4, treated with or without increasing amount of RSPO2 (c) or RSPO3 (d) overnight. Cells were starved 3-6 h before the stimulation. Ratio, relative levels of pSmad1 normalized to tSmad1. Representative data from two independent experiments are shown. e qRT-PCR analysis of BMP target ID1 in HEPG2 cells upon BMP4, with or without overnightRSPO2 treatment. $n=3$ experimentally independent samples. Data are displayed as means \pm SD. ${ }^{\star \star} P<0.01,{ }^{\star \star \star} P<0.001$ from two-tailed unpaired $t$-test. $\mathbf{f}$, $\mathbf{g} B R E$ reporter assay in HEPG2 cells upon siLRP5/6 and siLGR4/5 knockdowns, with or without overnight BMP4 and RSPO2 treatment as indicated. $n=3$ biologically independent samples. $\mathbf{h}$ BRE reporter assay in HEPG2 cells stimulated overnight by BMP4 with or without increasing amount of WNT3A treatment. WNT3A activity was validated in Supplementary Fig. 1b. $n=3$ biologically independent samples. i Domain structures of RSPO2 and deletion mutants used in $\mathbf{j}$. sp, signal peptide; FU, furin domain; TSP1, thrombospondin domain 1. $\mathbf{j}$ BRE reporter assay in HEPG2 cells stimulated overnight with BMP4, and with or without RSPO2 WT or FU1/2 or TSP1 deletion mutants, respectively. $n=3$ biologically independent samples. Data for reporter assays (a, $\mathbf{b}, \mathbf{f}-\mathbf{h}, \mathbf{j})$ are displayed as means \pm SD, and show a representative of multiple independent experiments. ns, not significant; ${ }^{\star} P<0.05,{ }^{\star \star} P<0.01,{ }^{\star \star \star} P<0.001$, and ${ }^{\star \star \star \star} P P<0.0001$ from two-tailed unpaired $t$-test $(\mathbf{a}, \mathbf{b}, \mathbf{f}, \mathbf{g}$, j) or one-way ANOVA with Dunnett test (h). $\mathbf{k}$, I Western blot analyses of phosphorylated Smad1 (pSmad1) and total Smad1 (tSmad1) in $\mathrm{H} 1581$ cells upon siRNA transfection as indicated, with $0 \mathrm{~h}, 1 \mathrm{~h}$, and $2 \mathrm{~h}$ of BMP4 stimulation. Ratio, relative levels of pSmad1 normalized to $\mathrm{tSmad1}$. Representative data from two independent experiments are shown.

glands ${ }^{35-37}$. In contrast, overexpression of $r s p o 2$ failed to induce enlarged heads but instead induced spina bifida with reduced head structures, yielding the first indication that $r s p o 2$ does not act by the common mode of sequestering BMP ligands (Supplementary Fig. 2c).
To confirm the rspo2 morpholino data, we used a previously established guide RNA (gRNA)27 to generate Crispr-Cas9mediated Xenopus rspo2 knockout (KO) embryos (Supplementary Fig. 3a-e). We then established gRNAs to generate Crispr-Cas9 mediated knockouts of the BMP antagonists chordin (chd) and 


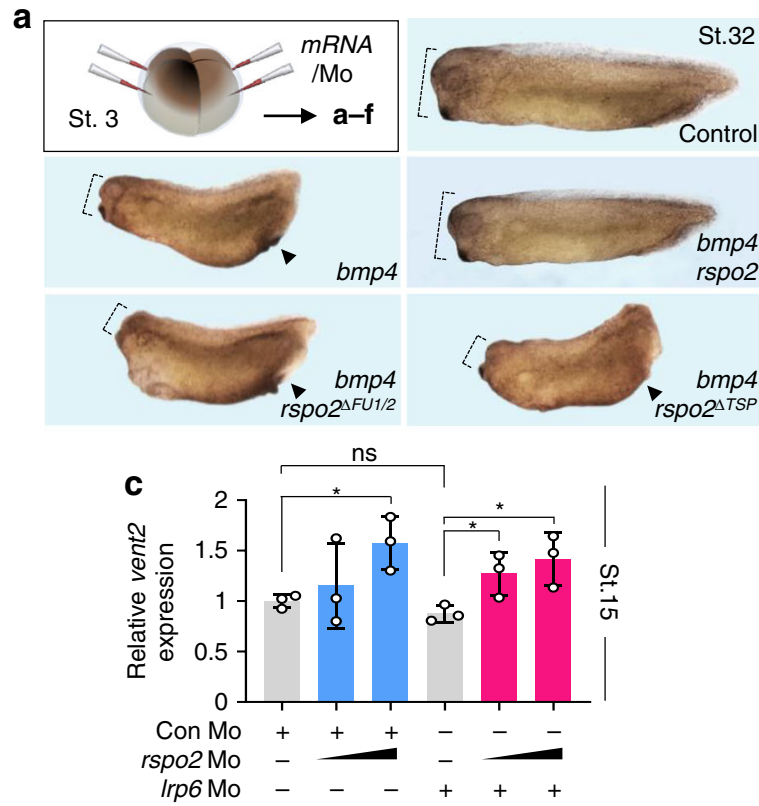

b
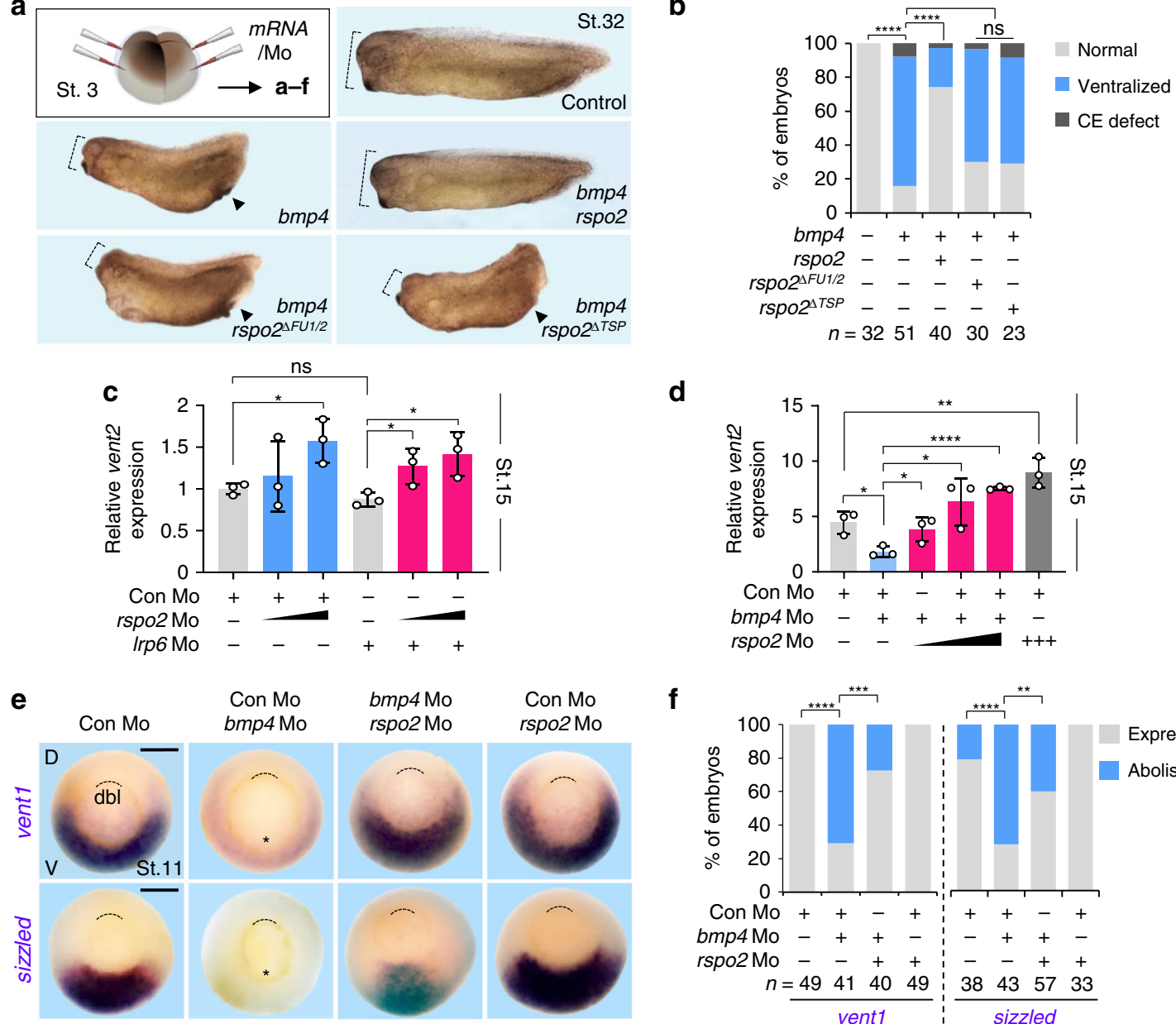

d
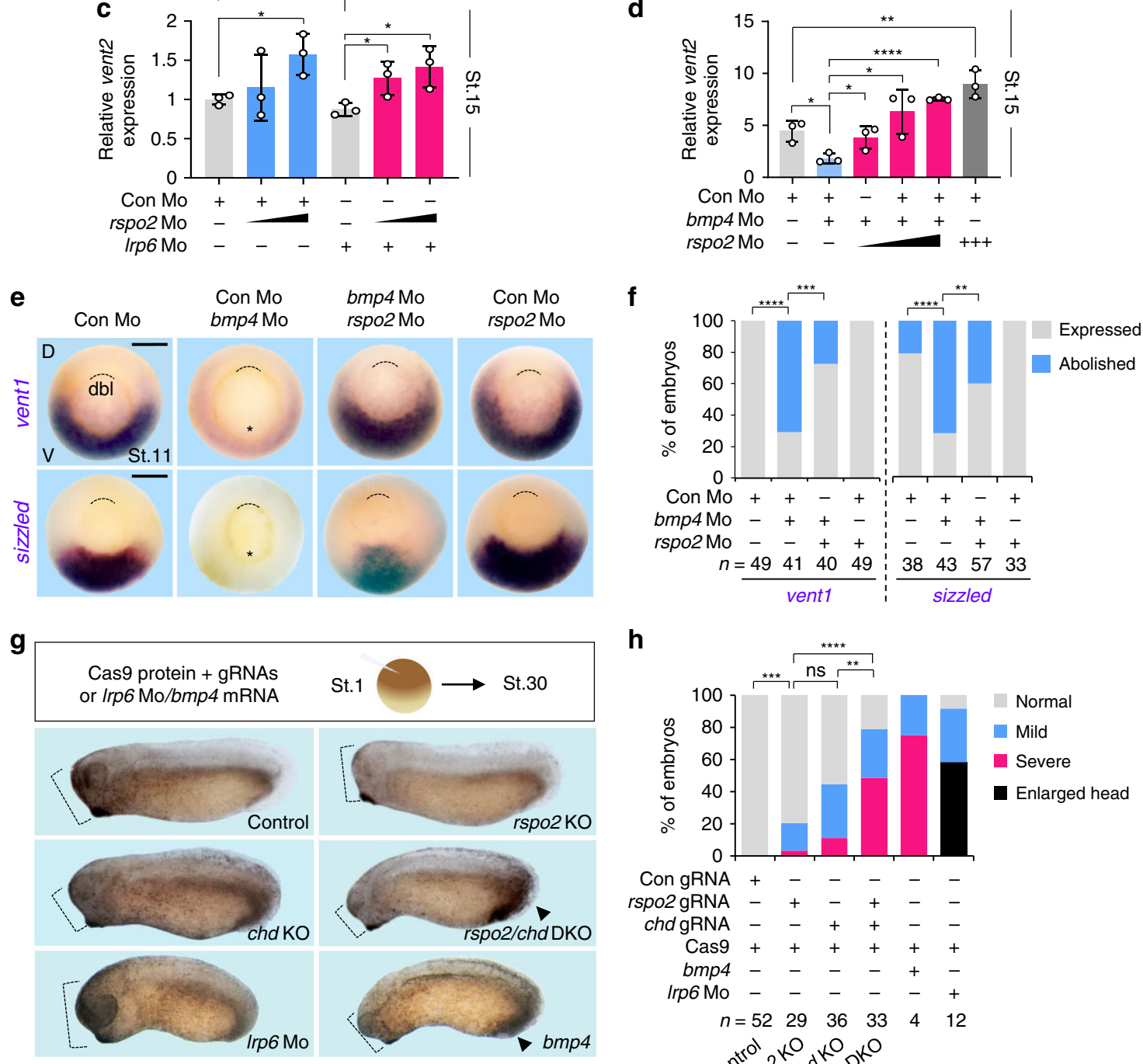

$\mathbf{h}$

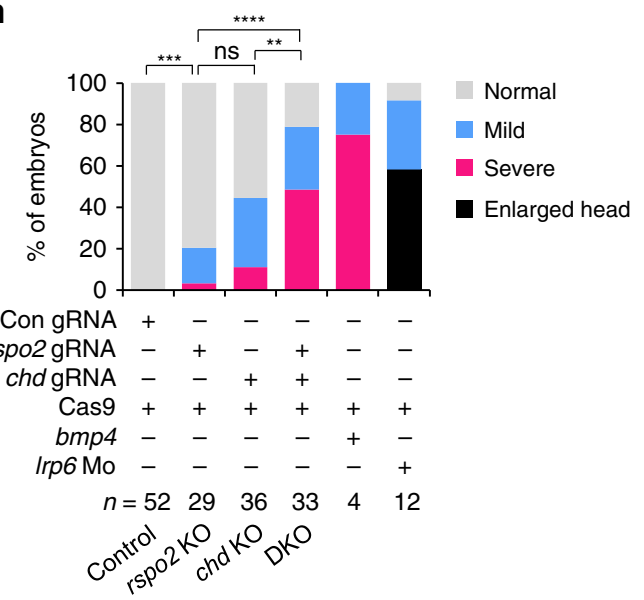

noggin (nog) (Supplementary Fig. 3a-e), whose microinjection with Cas9 protein yielded mildly ventralized embryos, which were rescued by chordin or noggin DNA, validating the specificity of the gRNAs (Supplementary Fig. 3f-i). Injection of rspo2 gRNA with Cas9 protein resulted in mildly ventralized embryos (Fig. $2 \mathrm{~g}$, h, Supplementary Fig. 4a, b) and increased BMP target gene (sizzled, vent1) expression, similar to knockouts of chordin or noggin (Supplementary Fig. 4c, f). Importantly, combined injection of rspo2 gRNA with either chordin or noggin gRNAs yielded strongly ventralized embryos (Fig. 2g, h, Supplementary Fig. 4a, b) and hyperactivated BMP signaling (Supplementary Fig. 4c-f). Moreover, injection of rspo3 mRNA rescued bmp4-mediated increase of sizzled expression, suggesting that overexpressed rspo3 is also able to antagonize BMP signaling in Xenopus (Supplementary Fig. 4g, h), as in HEPG2 cells (Fig. 1a). We conclude that rspo2 is required to antagonize BMP signaling and acts in concert with BMP antagonists for proper axial patterning during Xenopus embryogenesis.

Rspo2 is a negative feedback regulator in the Xenopus BMP4 synexpression group. In early vertebrate embryos, genes belonging to certain signaling networks form characteristic synexpression groups, i.e., genetic modules composed of genes 
Fig. 2 Rspo2 inhibits BMP4 signaling in Xenopus dorsoventral embryonic patterning. a Microinjection strategy for a-f, and representative phenotypes of Xenopus laevis tadpoles (St. 32) injected with the indicated mRNAs radially at 4-cell stage. Dashed lines, head size. Arrowheads, enlarged ventral structure. b Quantification of embryonic phenotypes shown in a. 'Ventralized' represents embryos with both small head and enlarged ventral structure, reminiscent of BMP hyperactivation. 'CE defect' refers to embryos with convergent extension (gastrulation) defects, unrelated to BMP signaling. Note that rspo2 mRNA dosage used in a was below those that cause gastrulation defects. $n=$ number of embryos. c, $\mathbf{d}$ BMP-(vent2) reporter assays with Xenopus laevis neurulae (St.15) injected with reporter plasmids and the indicated Mo at 4-cell stage. $n=$ biologically independent samples and data are displayed as means \pm SD ns, not significant. ${ }^{\star} P<0.1,{ }^{\star \star} P<0.01,{ }^{\star \star \star \star} P<0.0001$ from two-tailed unpaired $t$-test. e In situ hybridization of vent1 and sizzled in $X$ enopus laevis gastrulae (St.11, dorsal to the top, vegetal view) injected as indicated. D, dorsal, V, ventral. Asterisk, abolishment of the expression. Dashed line, dorsal blastopore lip (dbl). Scale bar, $0.5 \mathrm{~mm}$. f Quantification of embryonic phenotypes shown in (e). 'Expressed', normal, increased or reappearance of vent1/sizzled expression. 'Abolished', complete absence of vent1/sizzled expression. Data are pooled from two independent experiments. $n=$ number of embryos. $\mathbf{g}$ Microinjection strategy and representative phenotypes of Xenopus tropicalis tadpole (St.30) Crispants and tadpoles (St.30) injected with bmp4 mRNA or Irp6 Mo. At 1-cell stage, Cas9 protein with guide RNA (gRNA) targeting rspo2 or chd, or both gRNAs were injected animally. Dashed lines, head size. Arrowheads, enlarged ventral structure. $\mathbf{h}$ Quantification embryonic phenotypes shown in $\mathbf{g}$. 'Severe' showed small head, enlarged ventral tissues and short body axis. 'Mild' showed one or two of the defects described above. 'Normal' showed no visible differences to the uninjected control. $n=$ number of embryos. ns, not significant. ${ }^{\star \star} P<0.01,{ }^{\star \star \star} P<0.001,{ }^{\star \star \star \star} P<0.0001$ from two-tailed $\chi 2$ test comparing normal versus ventralized phenotypes (b), twotailed $\chi 2$ test comparing expressed versus abolished (f), or two-tailed $\chi 2$ test comparing normal versus severe and mild defects $\mathbf{h}$.

that show tight spatio-temporal RNA coexpression and that function in the respective signaling pathway ${ }^{40}$. A wellcharacterized example is the BMP4 synexpression group, members of which are expressed like this growth factor-dorsally in the eye, heart and proctodeum of tailbud stage Xenopus embryos (Fig. 3a). This group consists of at least eight members, which all encode positive or negative feedback components of the BMP signaling cascade as studied in early development, including ligands, receptors and downstream components of the pathway ${ }^{41}$. Interestingly, we found that rspo2 is part of the BMP4 synexpression group, being coexpressed with bmp4 from gastrula to tadpole stages (Fig. 3a), suggesting that its expression depends on BMP signaling as for other synexpressed genes. To test this idea, we employed Xenopus animal cap explants, which express low levels of rspo 2 and $b m p 4$ to monitor $r s p o 2$ induction upon $b m p 4$ overexpression (Fig. 3b). Indeed, bmp4 induced rspo 2 expression by qRT-PCR (Fig. 3c) and in situ hybridization (Fig. 3d, e), similar to bmp4 direct targets sizzled (Fig. 3c-e) and vent1 (Fig. 3c). To test whether rspo 2 is an immediate early target of BMP4, we blocked protein synthesis with cycloheximide $(\mathrm{CHX})^{41}$. Interestingly, while induction of the direct BMP4 targets sizzled and vent 1 by bmp4 was unaffected by $\mathrm{CHX}$, rspo 2 induction was inhibited (Fig. 3b-e). We conclude that rspo2 is a negative feedback inhibitor within the BMP4 synexpression group and that it is an indirect BMP target gene, whose expression may depend on transcription factors of the e.g., Vent or Msx families $^{41,42}$ (Fig. 3f).

RSPO2 and RSPO3 bind BMPR1A via the TSP1 domain to antagonize BMP signaling. Given that RSPOs act by promoting receptor endocytosis ${ }^{14,17}$, we postulated that RSPO2 might regulate BMP signaling through its receptors: ACVR1, BMPR1A and BMPR1B. To test this hypothesis, we analyzed the effect of RSPO14 treatment on BMP signaling induced by constitutively active ACVR1/BMPR1A/BMPR1B (ACVR1/BMPR1A/BMPR1BQD). Interestingly, RSPO2 and RSPO3 treatment specifically inhibited BMPR1AQD but not ACVR1QD or BMPR1BQD, while RSPO1 and $\mathrm{RSPO} 4$ had no effect to any of the constitutively active receptors (Fig. 4a-c).

Indeed, cell surface binding assay and in vitro binding assay revealed that RSPO2 and RSPO3, but not RSPO1 and RSPO4, bound the extracellular domain (ECD) of BMPR1A (Fig. 4d, e, Supplementary Fig. 5a). RSPO2 showed high affinity with BMPR1A ECD $\left(K_{\mathrm{d}} \approx 4.8 \mathrm{nM}\right)$ (Fig. $\left.4 \mathrm{f}\right)$, comparable to the RSPO-LGR interaction ${ }^{24}$. To further delineate the domains required for BMPR1A binding, we analyzed deletion mutants of
RSPO2 in cell surface binding assays with BMPR1A ECD, and found BMPR1A binding required the TSP1 but not the FU domains of RSPO2, while, conversely, LGR binding required the FU domains but not TSP1 (Supplementary Fig. 5b, c). The importance of the TSP1 domain was confirmed by in vitro binding assay showing that the isolated TSP1 domain of RSPO2, but not RSPO1, was sufficient to interact directly with BMPR1A ECD (Fig. 4g, h). Similarly, BMPR1A binding required the TSP1 domain also in RSPO3, suggesting that an analogous mode of binding applies to RSPO2 and RSPO3 (Supplementary Fig. 5d, e). Our results indicate that the specificity for the RSPO-BMPR1A interaction resides in the TSP1 domain of RSPOs. Consistently, the RSPO1 TSP1 domain shows only 43 and 50\% sequence similarity to RSPO2 and RSPO3, respectively ${ }^{28}$. We next asked whether TSP1-domain swapping could convey BMP signaling inhibition to RSPO1. To this end, we generated a RSPO1 chimera (R1-TSPR2) possessing the TSP1 domain of RSPO2 (Fig. 4i). R1$\mathrm{TSP}^{\mathrm{R} 2}$ activated WNT signaling (Fig. 4j) and interacted with LGR4 (Supplementary Fig. 5f). However, unlike wild-type RSPO1, R1-TSPR2 bound to BMPR1A (Supplementary Fig. 5f) and antagonized BMP signaling, mimicking the effects of RSPO2 (Fig. 4k).

The importance of the TSP1 domain in BMP inhibition was further corroborated in Xenopus, where we took advantage of the fact that the TSP1-domain is encoded by a distinct exon in the $3^{\prime}$ end of the $r s p o 2$ gene. We generated a rspo $2 \mathrm{Mo}\left(r s p o 2^{\triangle T S P} \mathrm{Mo}\right)$, which specifically abolished TSP1-domain splicing, yielding 3' truncated rspo 2 mRNA lacking the TSP1 domain but retaining the FU domains (Fig. 5a). Microinjection of $r s p o 2^{\Delta T S P}$ Mo resulted in ventralized tadpoles with shorter axis and reduced heads compared to control tadpoles, which was partially rescued by introducing a non-targeted rspo2 mRNA (Supplementary Fig. 6a, b). rspo $2^{\triangle T S P}$ Morphants had no effect on WNT signaling (Fig. 5b), confirming that it does not interfere with Rspo2 FU domains that are essential for WNT activation. However, rspo $2^{\triangle T S P}$ Mo increased BMP signaling (Fig. 5c). Similar to chordin and rspo2 Morphants, rspo2 $2^{\Delta T S P}$ Morphants showed expanded expression of the BMP target genes vent 1 and sizzled in gastrulae (Fig. 5d, e, Supplementary Fig. 6c, d) ${ }^{38}$, and corresponding tadpoles were ventralized, displaying decreased $b f 1$ and $m y o D$ and increased sizzled expression (Fig. 5f, g) ${ }^{38}$. Coexpression of dominant negative bmprla (bmprla $\left.{ }^{D N}\right)$ rescued these defects (Fig. 5d, g, Supplementary Fig. 6c, d). Taken together, these results emphasize that the TSP1 domain is a key element in providing target specificity to RSPOs, both in vitro and in vivo, and that it dictates their BMP-inhibitory function. 
a

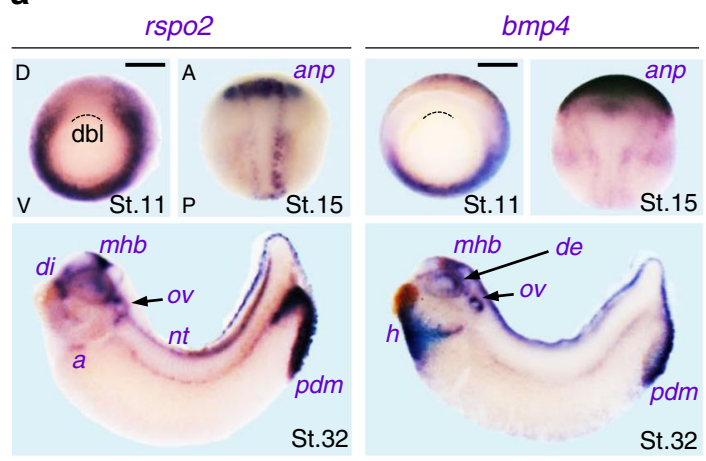

b
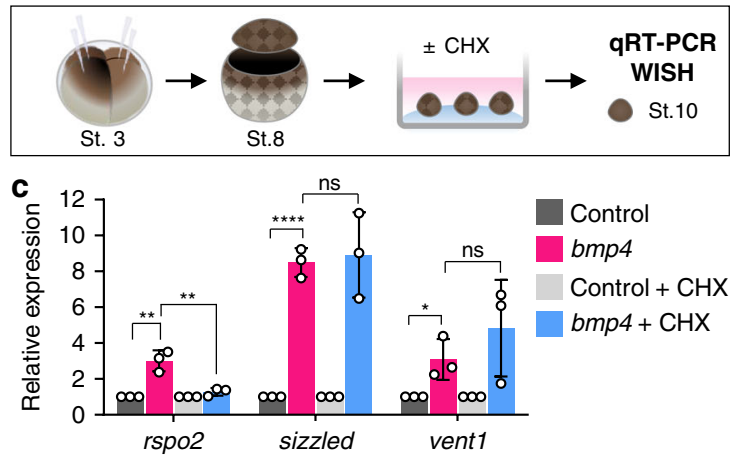

d

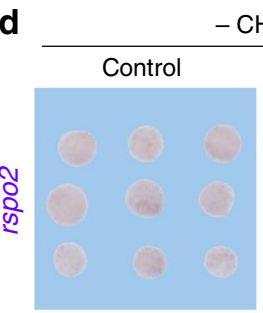
$-\mathrm{CHX}$ bmp4

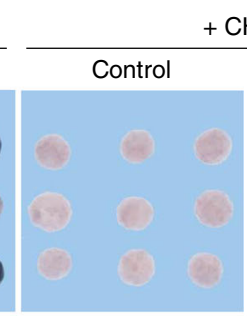
$\mathrm{CHX}$

:::
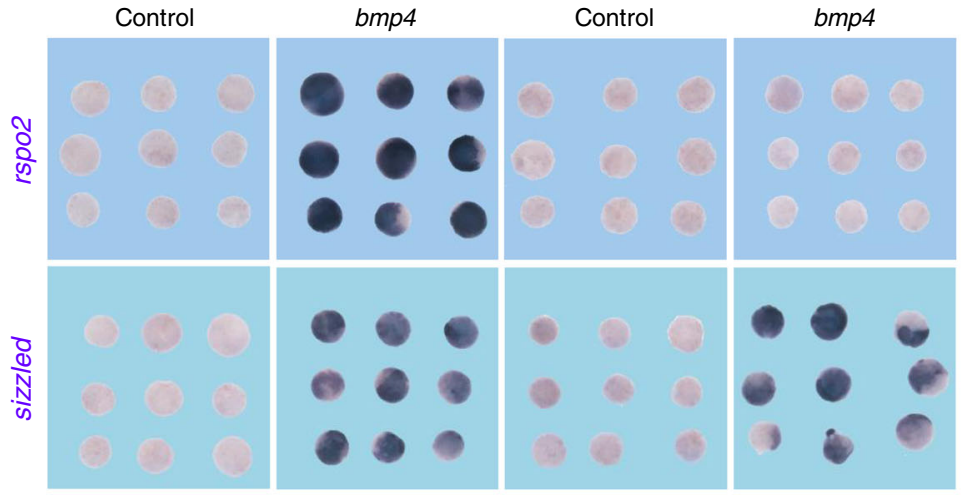

e

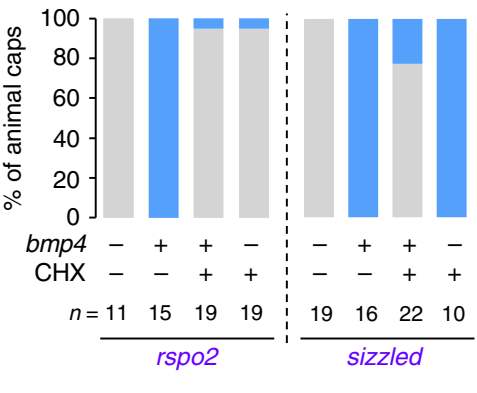

(-) rspo2/szl

(+) rspo2/szl

f

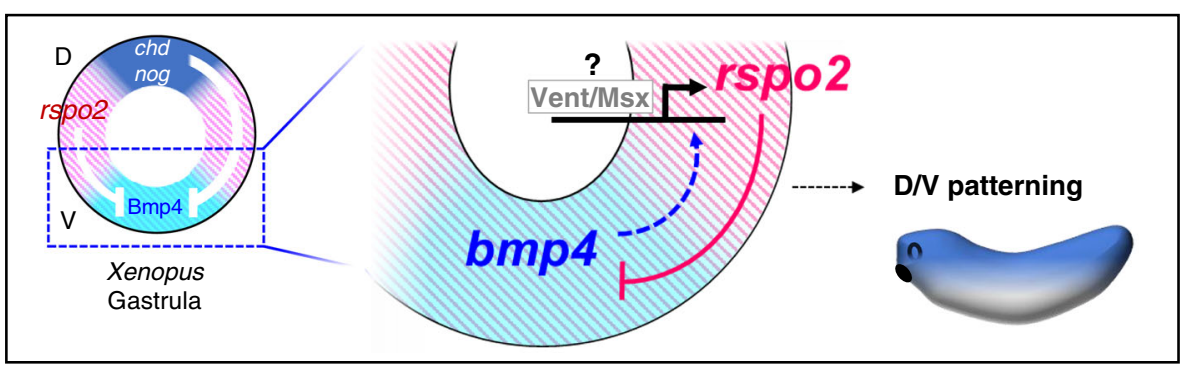

Fig. 3 Rspo2 is a negative feedback inhibitor in the BMP4 synexpression group. a In situ hybridization of rspo2 and bmp4 in Xenopus laevis at gastrula (St. 11, dorsal to the top, vegetal view), neurula (St. 15, anterior to the top, dorsal view), and tadpole (St. 32, anterior to the left, lateral view). Dashed lines, dorsal blastopore lip (dbl); anp, anterior neural plate; di, diencephalon; mhb, mid-hindbrain boundary; ov, otic vesicle; a, atria; nt, neural tube; de, dorsal eye; h, heart; pdm, proctodeum. Scale bar, $0.5 \mathrm{~mm}$. b Microinjection and experimental scheme for $\mathbf{c}-\mathbf{e}$. 2 or 4 cell stage Xenopus laevis embryos were animally injected with control ( $p p l)$ or bmp4 mRNA. The animal cap (AC) explants were dissected from injected embryos at stage 8 , and either treated or untreated with cycloheximide (CHX) until control embryos reached stage 10 for qRT-PCR (c) or in situ hybridization (d, e). c qRT-PCR of rspo2, sizzled, and vent1 expression in the AC explants injected and treated as indicated. Data are pooled from three independent experiments with similar results and displayed as means \pm SD. ns, not significant, ${ }^{\star} P<0.05,{ }^{\star \star} P<0.01,{ }^{\star \star \star \star} P<0.0001$ from two-tailed unpaired $t$-test. $\mathbf{d}$ In situ hybridization of $r s p 02$ and sizzled in the AC explants injected and treated as indicated. e Quantification of (d). $n=$ number of the AC explants. $\mathbf{f}$ Model for Rspo 2 function as a negative feedback inhibitor of BMP4 in Xenopus dorsoventral patterning.

RSPO2 destabilizes the BMP receptor BMPR1A. To investigate the consequence of RSPO-BMPR1A binding, we monitored BMPR1A protein levels upon RSPO2 knockdown in H1581 cells and found that siRSPO2 treatment increased BMPR1A protein levels (Fig. 6a). Similarly in Xenopus whole embryos, microinjection of mRNA encoding $r s p o 2$ but not $r s p o 2^{\Delta \mathrm{FU} 1 / 2}$ or rspo $2^{\Delta \mathrm{TSP}}$ decreased protein levels from coinjected bmprla-EYFP mRNA (Fig. 6b). Immunofluorescence microscopy (IF) of Xenopus animal cap explants showed that Bmprla-EYFP localizes to the plasma membrane, where it was once again reduced by rspo2 but not by $r s p o 2^{\Delta \mathrm{FU} 1 / 2}$ or $r s p o 2^{\Delta \mathrm{TSP}}$ mRNA (Fig. $6 \mathrm{c}-\mathrm{e}$ ). Focusing on Xenopus ventrolateral marginal zone (VLMZ) explants, where endogenous rspo2, bmprla and bmp4 are coexpressed, showed that ablation of rspo2 by Mo injection results in significant increase of Bmprla-EYFP plasma membrane levels (Fig. $6 \mathrm{f}-\mathrm{h}$ ). Moreover, in VLMZ from rspo $2^{\Delta \mathrm{TSP}}$ Morphants, Bmprla levels were also increased (Fig. $6 \mathrm{f}-\mathrm{h}$ ), which was confirmed by western blot analysis (Fig. 6i). Altogether, our results suggest that RSPO2 destabilizes BMPR1A.

RSPO2 requires ZNRF3 to antagonize BMP receptor signaling. We next turned to the role of the FU domains in RSPO2, which are also required for inhibition of BMP signaling (Figs. 1j, 2a, b and $6 \mathrm{~b}-\mathrm{e})$. FU1 and FU2 domains confer RSPO binding to ZNRF3/RNF43 and LGRs, respectively ${ }^{27}$. Since our results demonstrated an LGR-independent mode of action (Fig. 1f), and 
a

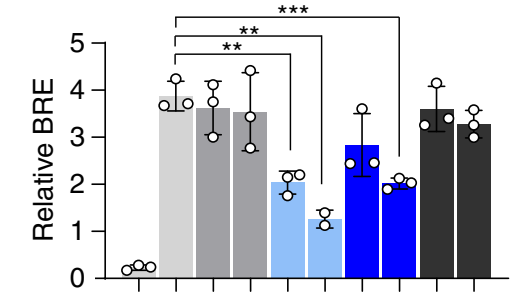

$\mathrm{BMPR}^{\mathrm{BQD}}-+++++++++$

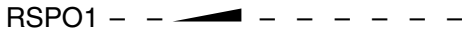

$\mathrm{RSPO} 2----\infty-$

$\mathrm{RSPO} 3---\mathrm{C}_{-}-$

RSPO4 - b

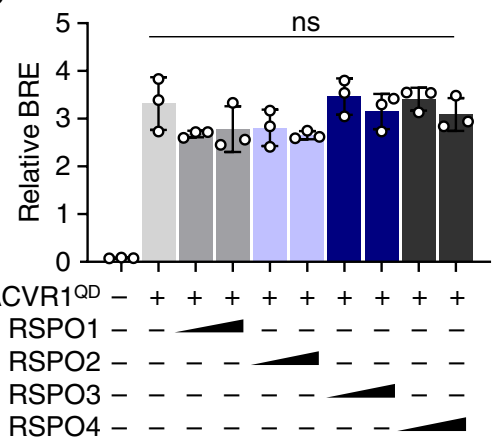

C

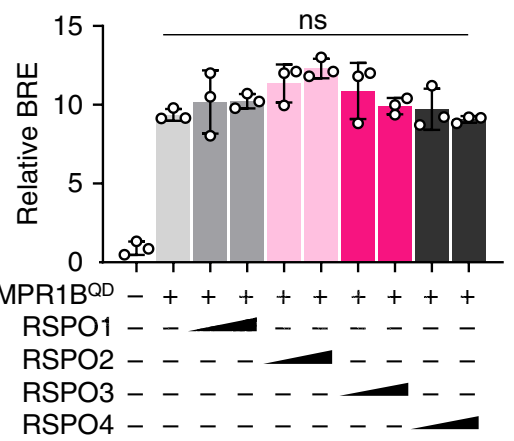

d
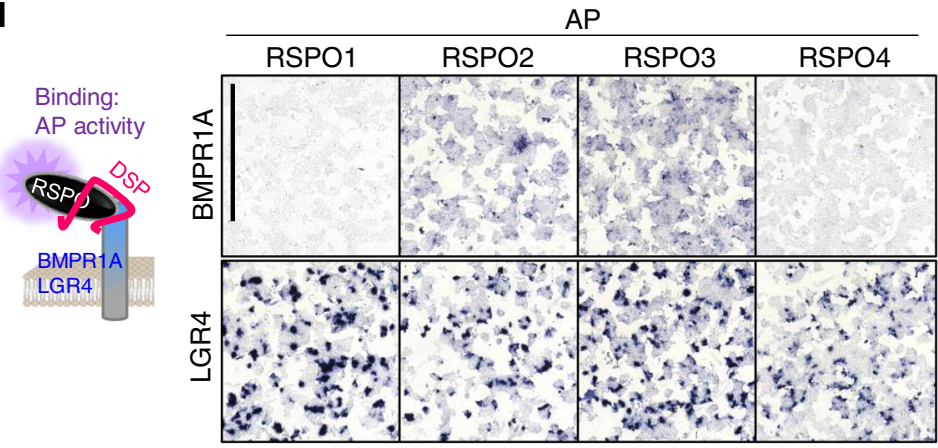

e

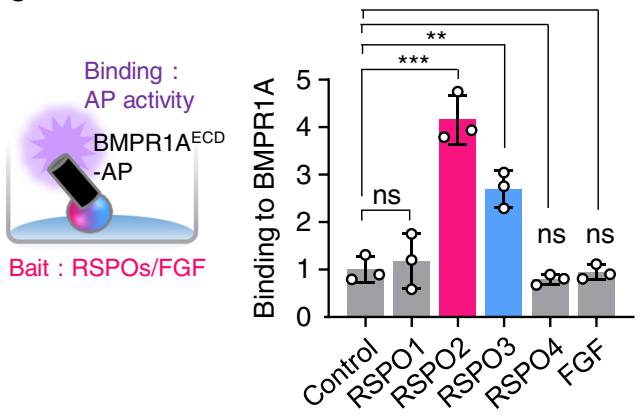

h

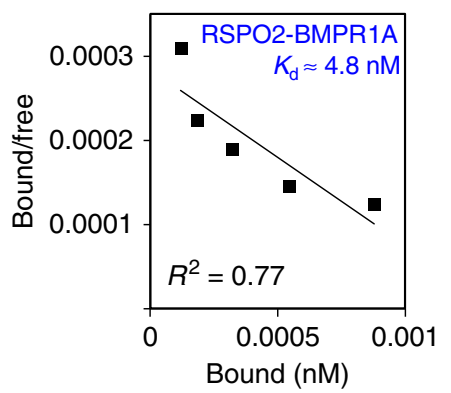

g
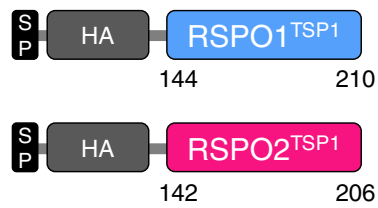

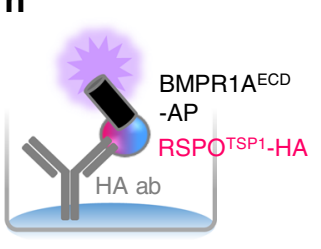

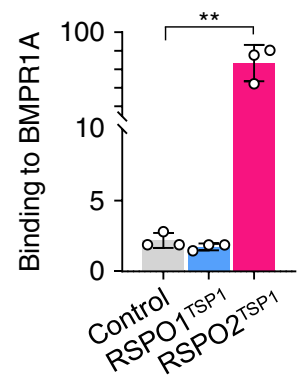

i

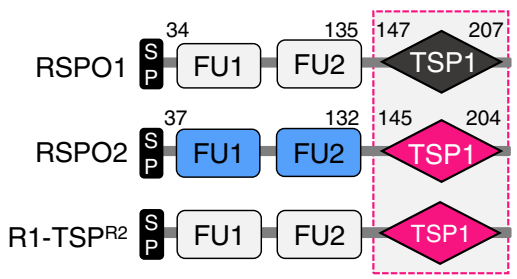

j

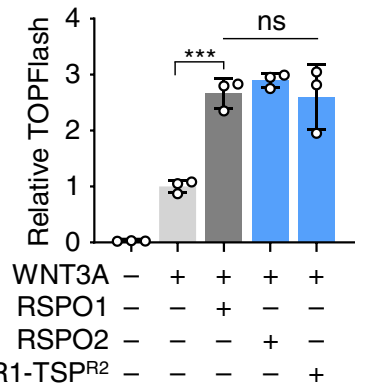

k

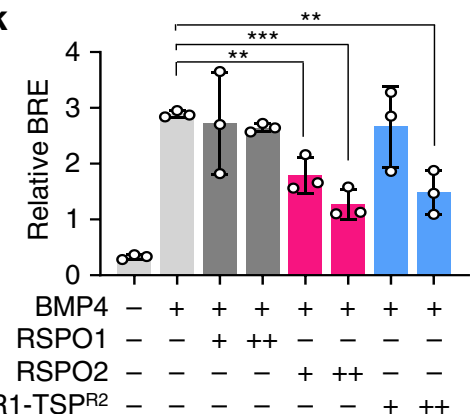

since $r s p o 2$ destabilized Bmprla (Fig. 6b), we hypothesized that RSPO2 acts via ZNRF3/RNF43 E3 ligases to interfere with BMPR1A. ZNRF3 and RNF43 were both expressed in HEPG2 and H1581 cells, and could be significantly knocked down by siRNA (Supplementary Fig. 7a). Knockdown of ZNRF3/RNF43 (Fig. 7a) or expression of a dominant negative ZNRF3 $\left(\mathrm{ZNRF} 3^{\triangle \mathrm{R}}\right)^{26}$ (Fig. $7 \mathrm{~b}$ ) prevented inhibition of BMP signaling by RSPO2 in HEPG2 cells, supporting that RSPO2 requires ZNRF3/ RNF43 to antagonize BMP signaling. In Xenopus, znrf3 was broadly expressed from gastrula stages onwards, like bmprla (Supplementary Fig. 7b, c). znrf3 ablation by Mo elicited head and axis defects that were rescued by coinjection of human ZNRF3 mRNA, as previously described ${ }^{43}$ (Supplementary Fig. 7d, e).
Interestingly, znrf3 Morphants at neurula showed increased BMP signaling by BMP-reporter assay and rspo2 mRNA coinjection could not reduce it (Fig. 7c). Moreover, IF in Xenopus animal cap explants showed that rspo2-induced destabilization of Bmprla protein levels was prevented by $Z N R F 3^{\triangle \mathrm{R}}$ (Fig. 7d, e). Altogether, these results support that to function as BMP antagonist, RSPO2 requires ZNRF3.

RSPO2 requires the FU1 but not FU2 domain to antagonize BMP signaling. To corroborate that to function as BMP antagonist, RSPO2 depends on ZNRF3/RNF43, but not on LGRs, we next generated deletion mutants of the FU1 and FU2 domains in human RSPO2, which mediate binding to ZNRF3/RNF43 and 
Fig. 4 RSPO2 and RSPO3 interact with BMPR1A via the TSP1 domain. a-c BRE reporter assays in HEPG2 cells transfected with constitutively active (QD) BMPR1A (a), ACVR1 (b), or BMPR1B (c) with or without BMP4 and RSPO1-4 treatment overnight. d Cell surface binding assay in HEK293T cells. (Left) Scheme of the assay. Cells were transfected with BMPR1A or LGR4 DNA, and treated with same amount of RSPO1-4-AP upon DSP crosslinking as indicated. Binding was detected as purple stain on cell surface by chromogenic AP assay. (Right) Images of cells transfected and treated as indicated. Data shows a representative from four independent experiments. For quantification, see Supplementary Fig. $5 \mathrm{a}$. Scale bar, $1 \mathrm{~mm}$. e $\mathrm{ln}$ vitro binding assay between RSPO1-4, FGF and BMPR1AECD. (Left) Scheme of the assay. RSPOs and FGF recombinant proteins were coated on plate as baits, followed by BMPR1AECD AP treatment overnight. (Right) Bound BMPR1A $E C D$ was detected by chromogenic AP assay. Normalized AP activity with control treatment was set to 1. f Scatchard plot of RSPO2 and BMPR1A ECD binding to validate $K_{d}$ for RSPO2-BMPR1A. $\mathbf{g}$ Domain structures of the RSPO1 and RSPO2 ${ }^{\text {TSP1 }}$ with Strep-HA and flag tags used in $\mathbf{h}$. SP, signal peptide; TSP1, thrombospondin domain 1. $\mathbf{h}$ In vitro binding assay for RSPOTSP1 and BMPR1AECD. (Left) Scheme of the assay. HA-harboring RSPO1/2 ${ }^{\mathrm{TSP} 1}$ were captured to HA antibody coated plate, and BMPR1AECD-AP was treated overnight. (Right) Bound BMPR1A to $\mathrm{RSPO}^{\mathrm{TSP} 1}$ was detected with absorbance. i Domain structures of the RSPO1, RSPO2, and R1-TSPR2. SP, signal peptide; FU, furin domain; TSP1, thrombospondin domain 1. Dashed box indicates the TSP1 domain swapping. $\mathbf{j}$ TOPFlash reporter assay in HEPG2 cells upon WNT3A with or without (i) as indicated. $\mathbf{k}$ BRE reporter assay in HEPG2 cells upon BMP4 with or without (i) as indicated. For reporter assays (a-c, $\mathbf{j}, \mathbf{k}), n=3$ biologically independent samples; In vitro binding assays $(\mathbf{e}, \mathbf{h}), n=3$ experimentally independent samples. All data are displayed as mean \pm SD. ns, not significant, ${ }^{\star} P<0.05,{ }^{\star \star} P<$ $0.01,{ }^{\star \star \star} P<0.001,{ }^{\star \star \star \star} P<0.0001$ from two-tailed unpaired $t$-test $(\mathbf{a}, \mathbf{b}, \mathbf{e}, \mathbf{h}, \mathbf{j}, \mathbf{k})$ or one-way ANOVA test (c).

LGRs, respectively ${ }^{27}$ (Supplementary Fig. 8a). RSPO2 ${ }^{\Delta \mathrm{FU} 1}$ lost ZNRF3 binding (Supplementary Fig. 8b), yet it bound LGR4 (Supplementary Fig. 8c), but did not inhibit BMP4 signaling (Fig. 7f). Conversely, RSPO2 ${ }^{\triangle \mathrm{FU}} 2$ bound ZNRF3 but not to LGR4 (Supplementary Fig. 8b, c), yet it still antagonized BMP4 signaling (Fig. $7 \mathrm{~g}$ ). To corroborate LGR-independent function in vivo, we generated Xenopus Rspo $2^{\Delta \mathrm{FU} 1}$ and FU2 point mutant Rspo2 ${ }^{\mathrm{F} 107 \mathrm{E}}$ (Supplementary Fig. 8d) ${ }^{17}$, which displayed ZNRF3 and LGR4 binding characteristics like human RSPO2 mutants (Supplementary Fig. 8e, f). IF in Xenopus animal cap explants injected with bmpr1a-EYFP and either rspo 2 wildtype or rspo 2 mutants confirmed that FU1 but not FU2 deletion eliminates the ability of Rspo2 to remove plasma membrane Bmprla (Fig. 7h, i). Taken together, our results clearly indicate that the FU1 mediated ZNRF3/RNF43 binding is crucial while FU2 mediated LGR binding is dispensable for RSPO2 to antagonize BMP receptor signaling.

RSPO2 bridges BMPR1A and ZNRF3 and triggers BMP receptor clearance from the cell surface. The interaction of RSPO2 and RSPO3 with BMPR1A, as well as ZNRF3, suggested that R-spondins bridge both transmembrane proteins. In vitro binding assays (Fig. 8a, b) and colocalization by IF (Fig. 8c, d, Supplementary Fig. 9a, b), confirmed that ZNRF3 interacted with BMPR1A in the presence of RSPO2 or RSPO3 but not of RSPO1. Emphasizing once again the importance of the FU1 and TSP1 domains for this interaction, in vitro ZNRF3-BMPR1A-RSPO2 ternary complex formation was prevented by TSP1, FU1/2, or FU1 deletion (Supplementary Fig. 9c-g), whereas it remained intact upon FU2 deletion (Supplementary Fig. 9h).

Since ZNRF3/RNF43 eliminate WNT receptors from the cell surface by co-internalization and lysosomal degradation ${ }^{25,26}$, we considered an analogous function in BMPR1A turnover. We monitored BMPR1A localization by IF in H1581 cells and found that it was absent from the plasma membrane but abundantly colocalized with ZNRF3 in cytoplasmic vesicles (Fig. 8e, i), suggesting that it may be internalized by endogenous RSPO2. Indeed, upon knockdown of RSPO2, but not LRP6 or LGR4/5, BMPR1A accumulated at the plasma membrane (Fig. $8 \mathrm{f}-\mathrm{i}$ ). Importantly, IF (Fig. 8j-m) and cell surface biotinylation assays (Fig. 8n) showed that upon ZNRF3/RNF43 siRNA treatment, BMPR1A also accumulated at the plasma membrane.

To test if RSPO2/ZNRF3 target BMPR1A for endocytosis and lysosomal degradation, we treated cells with the clathrin inhibitor monodansylcadaverin (MDC), which eliminated inhibition of BMP signaling by RSPO2 (Fig. 8o). In addition, siRSPO2 abolished the colocalization of BMPR1A with the early endosome marker EEA1 (Fig. 8p-q) and lysosomal marker Lamp1
(Fig. 8r-s), suggesting that RSPO2 binding promotes BMPR1A internalization and degradation via ZNRF3 ternary complex formation. Consistently, $20 \mathrm{~min}$ exposure to RSPO2 increased internalized BMPR1A in cell surface biotinylation assays in H1581 cells (Supplementary Fig. 10a) and induced vesicular Bmprla-EYFP in Xenopus animal caps (Supplementary Fig. 10b, c). Taken together, our results support a model (Supplementary Fig. 10d) wherein RSPO2 bridges ZNRF3 and BMPR1A and routes the ternary complex towards clathrin-mediated endocytosis for lysosomal degradation, thereby antagonizing BMP signaling. We suggest that a similar mechanism applies to RSPO3 but not RSPO1 and RSPO4.

\section{Discussion}

The three main findings of our study are (i) the discovery Rspondins as BMP receptor antagonists, (ii) that RSPO2 depletes BMPR1A/ALK3 by engaging ZNRF3 for internalization and lysosomal degradation, and (iii) that in Xenopus, rspo2 is a negative feedback inhibitor of the BMP4 synexpression group, which cooperates with Spemann organizer effectors to inhibit BMP signaling during axis formation. Given the importance of RSPOs and BMPs as developmental regulators, as well as growth factors of normal and malignant stem cells, these conclusions have implications for development and cancer.

With regard to stem cells, R-spondins are a key ingredient of the culture media, which have made the organoid revolution possible $e^{21,22}$ and their rational use requires an understanding of their mechanism of action. For example, the fact that R-spondins inhibit BMP signaling may explain the reported non-equivalence of WNT and RSPO ligands in stem cells and development ${ }^{31-33}$. It may also explain their potency as stem cell growth factors, as e.g., intestinal stem cells requires both, WNT activation and BMP inhibition $^{21,22}$.

TGF $\beta$ growth factors play an eminent role in biology and medicine, and their receptor signaling is exquisitely regulated extracellularly with over 20 TGF $\beta$ antagonists, most of which antagonize signaling by ligand sequestration (e.g., Cerberus, Chordin, Follistatin, Gremlin, Noggin, and Sost) ${ }^{1,11}$. Two extracellular BMP receptor antagonists are known, BMP3 and Inhibin ${ }^{12,13}$. Both are TGF $\beta$ family members, whose unproductive binding to type II receptors prevents signal transmission. Relatedly, the BMP antagonist BAMBI is a BMP pseudoreceptor lacking kinase activity, which also leads to formation of a deadend complex with BMP receptors ${ }^{44}$. In contrast, RSPO2 and RSPO3 share no sequence homology with TGF $\beta$ family members, they inhibit type I instead of type II BMP receptors, and they do so by engaging the ZNRF3 E3 transmembrane ubiquitin ligase to internalize BMPR1A. RSPO2 thereby routes BMPR1A to 

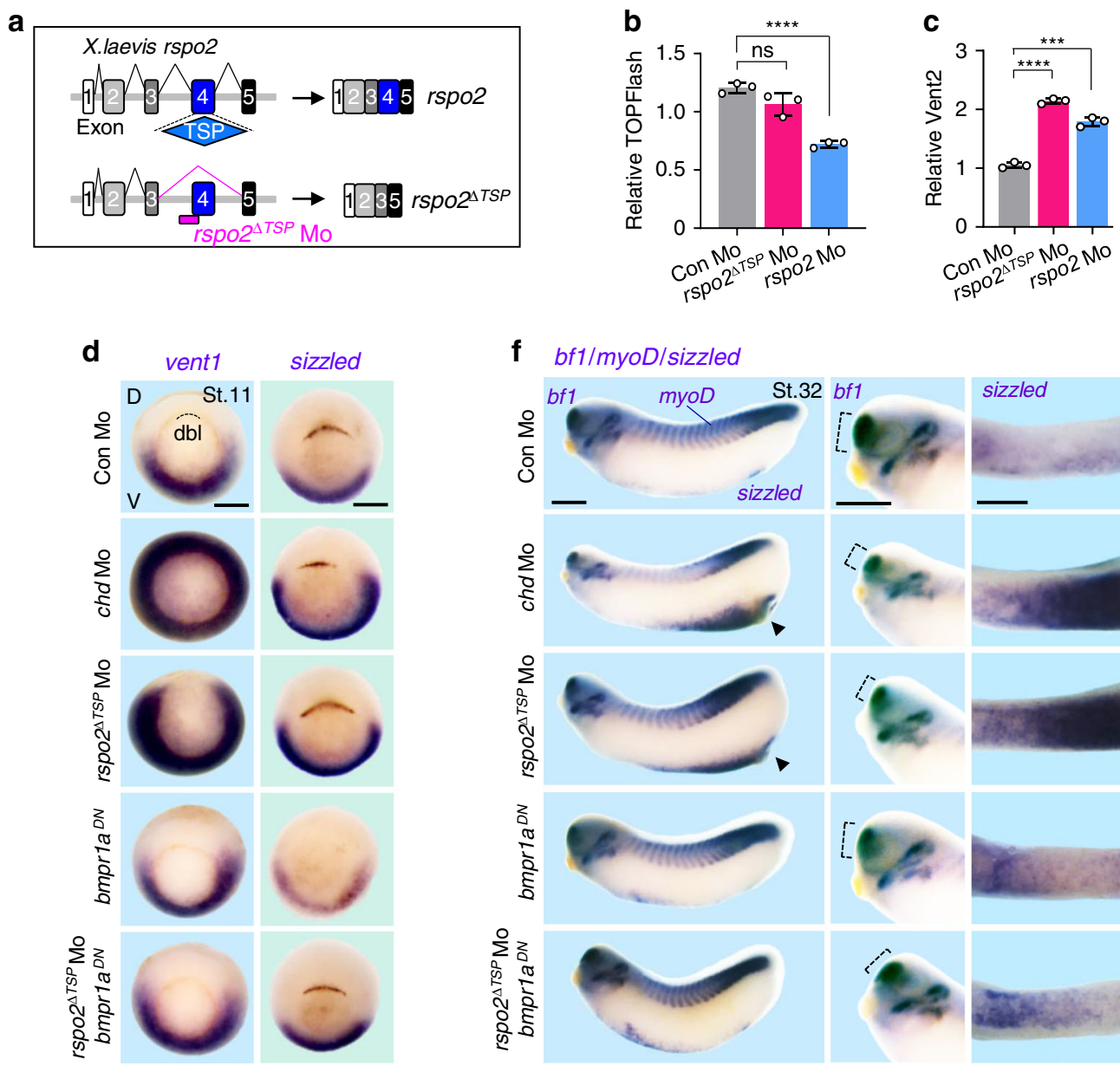

f $b f 1 / m y o D /$ sizzled
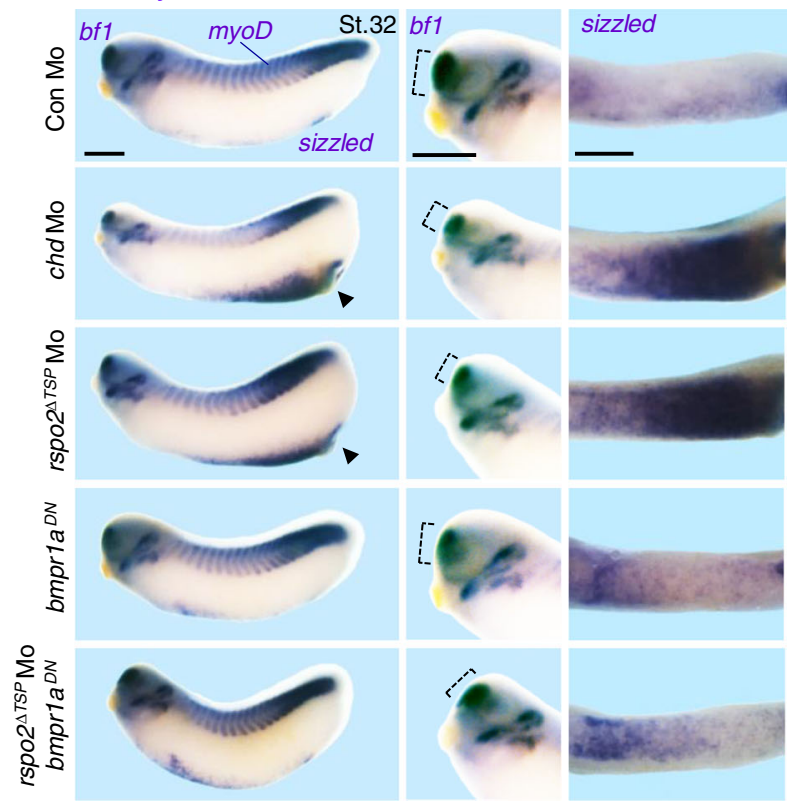

e

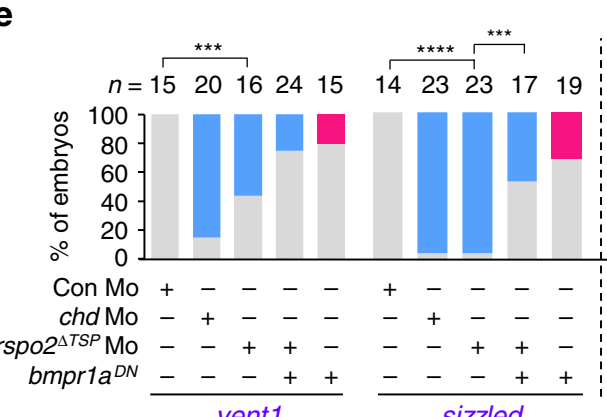

g

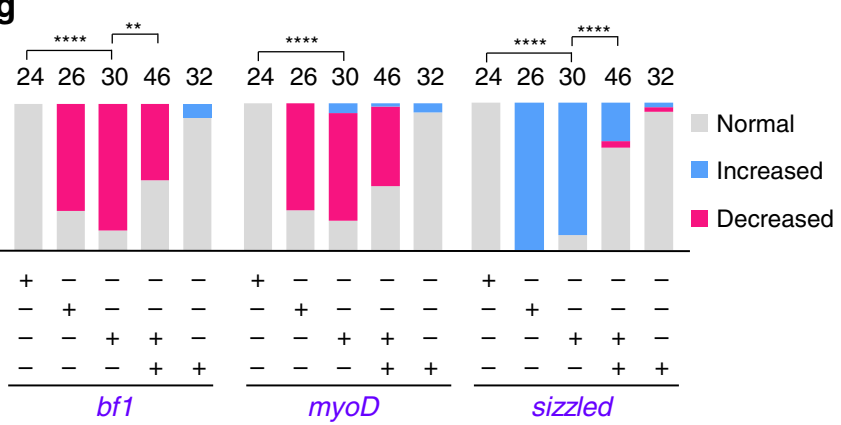

Fig. 5 Loss of Rspo2-TSP1 domain activates BMP signaling in Xenopus development. a Scheme for rspo $2^{\Delta T S P}$ splicing Mo in Xenopus laevis. b TOPFlash assay in Xenopus laevis neurulae (St.15) injected radially at 4-cell stage with reporter plasmids and Mo as indicated. Data are displayed as mean \pm SD; ns, not significant, ${ }^{\star \star \star \star} P<0.0001$ from two-tailed unpaired $t$-test. $n=3$ biologically independent samples. c BMP-reporter (vent2) assay in Xenopus laevis neurulae (St.15) injected radially at 4-cell stage with reporter plasmids and Mo as indicated. Data are displayed as mean \pm SD; ${ }^{\star \star \star} P<0.001,{ }^{\star \star \star \star} P<0.0001$ from two-tailed unpaired $t$-test. $n=3$ biologically independent samples. $\mathbf{d}$-g In situ hybridization of BMP4 targets vent1 and sizzled in Xenopus laevis. Embryos were injected radially and equatorially at 4-cell stage as indicated. Gastrulae (St.11) (d) and quantification (e); Tadpoles (St. 32) (f) and quantification (g). Dashed lines, dorsal blastopore lip (dbl) (d) or bf1 expression (e); D, dorsal; $V$, ventral. For f, left, lateral view; middle, magnified view of head; right, magnified view of ventral side. 'Increased/Decreased' represents embryos with significant expansion/reduction of sizzled or vent1 signals toward the dorsal/ventral side of the embryo (e), or with significant increase/decrease of the signal strength (g). ns, not significant. n, number of embryos. Scale bar, $0.5 \mathrm{~mm}$. Scoring of the embryos for quantification was executed with blinding from two individuals. For $\mathbf{e}, \mathbf{g}$, ${ }^{\star \star} P<0.01,{ }^{\star \star \star} P<0.001,{ }^{\star \star \star \star} P<$ 0.0001 from two-tailed $\chi 2$ test comparing normal versus increased. Data are pooled from at least two independent experiments.

clathrin-mediated endocytosis for lysosomal degradation. This mode of action resembles the function of the Spastic Paraplegia related gene NIPA1, a transmembrane antagonist, which promotes BMP receptor type II endocytosis and lysosomal degradation ${ }^{45}$.
Other type I BMP receptors besides BMPR1A include ACVRL1, ACVR1, and BMPR1B ${ }^{7}$. However, we found that RSPO2 specifically binds to BMPR1A but not to ACVR1 or BMPR1B (Supplementary Fig. 11), which explains why ACVR1 and BMPR1B signaling were not antagonized by RSPO2 in 
a

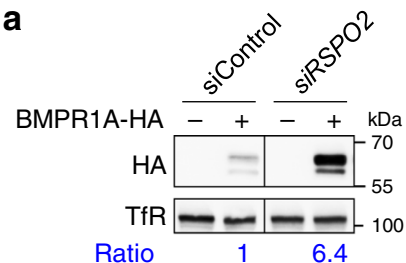

b

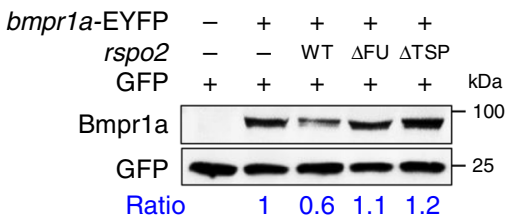

e
C

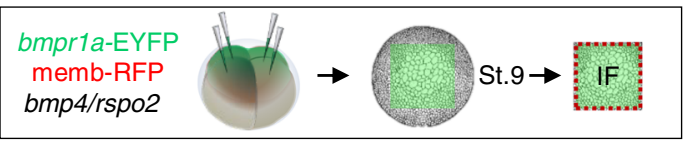

d

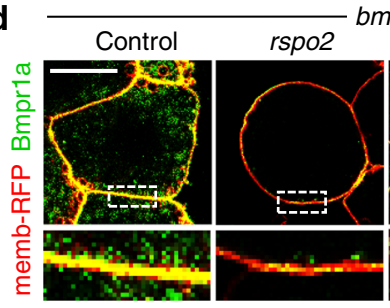

$\mathbf{f}$

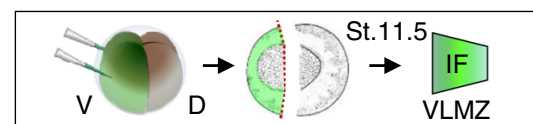

g

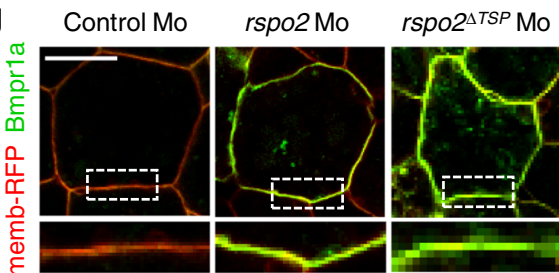

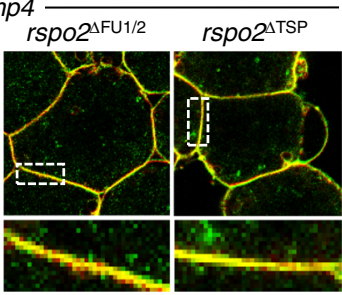

h

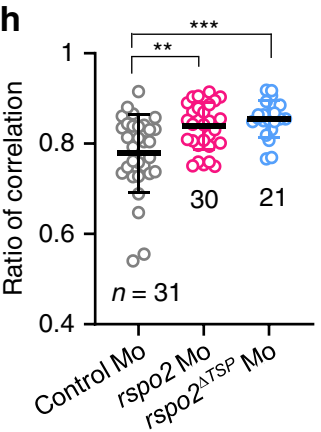

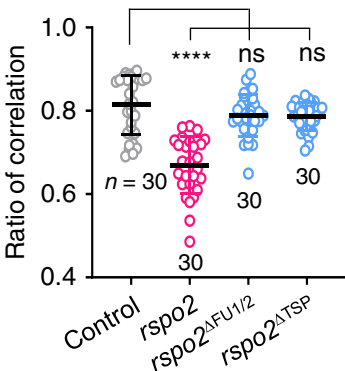

i bmpr1a-EYFP Control Mo rspo2 Mo rspo $2^{\triangle T S P}$ Mo Bmpr1a Gapdh

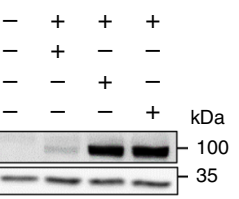

Fig. 6 RSPO2 removes cell surface BMPR1A. a Western blot analysis in $\mathrm{H} 1581$ cells treated with siControl or siRSPO2 as indicated and transfected with or without BMPR1A-HA DNA. Transferrin receptor (TfR), a loading control. Ratio, relative levels of BMPR1A-HA normalized to TfR. Data shows representative result from 3 independent experiments with similar conclusion. b Western blot analysis of Bmpr1a in Xenopus laevis (St. 15) neurulae injected animally at 2cell to 4-cell stages as indicated. GFP mRNA was injected as an injection control. Ratio, relative levels of Bmprla normalized to GFP. Data shows representative result from 3 independent experiments with similar conclusion. c Scheme for immunofluorescence microscopy (IF) in Xenopus laevis animal cap (AC) explants. Embryos were injected animally at 4-cell stage with bmpria-EYFP and memb-RFP mRNA along with bmp4 and rspo2 wild-type or mutant mRNA. AC explants were dissected at St.9 for IF. Membrane (memb)-RFP was used as a control comparing relative change of Bmprla-EYFP signal at cell surface. d IF for Bmpr1a (green) and cell membrane (red) in AC explants injected as indicated, with a representative cell (top) and magnification (inset). Scale bar, $20 \mu \mathrm{m}$. e Quantification of $\mathbf{d}$. $n=$ the number of areas analyzed and data are displayed as mean \pm SD. ns, not significant; ${ }^{\star \star \star \star} P<0.0001$ from two-tailed unpaired $t$-test. $\mathbf{f}$ Scheme for IF in Xenopus laevis ventrolateral marginal zone explants (VLMZ). Embryos were ventrally injected at 4-cell stage with bmpr1a-EYFP and memb-RFP mRNA with Mo. VLMZs were dissected at stage 11.5 for IF. $\mathbf{g}$ IF for Bmpr1a (green) and cell membrane (memb-RFP, red) in VLMZ injected with mRNA and Mo as indicated. Scale bar, $20 \mu \mathrm{m}$. $\mathbf{h}$ Quantification of $\mathbf{g} . n=$ the number of areas analyzed and data are displayed as mean \pm SD. ${ }^{\star \star} P<0.01,{ }^{\star \star \star} P<0.001$ from two-tailed unpaired $t$-test. i Western blot analysis of Bmprla in Xenopus laevis neurulae (St. 18) injected radially at 4-cell stage as indicated. Gapdh, a loading control. Data shows representative result from 2 independent experiments with similar conclusion.

human cells (Fig. 4a-c). Consistently, BMPR1A and e.g., BMPR1B only show $42 \%$ identity in their extracellular domain ${ }^{46}$.

BMPR1A engages not only various BMPs but also GDFs ${ }^{1}$, and hence RSPO-mediated inhibition may potentially affect signaling in multiple contexts. On the other hand, the specificity of RSPO2 for BMPR1A may provide therapeutic opportunities on the background of pleiotropic BMP ligands effects.

RSPO2 engages ZNRF3 to antagonize BMP signaling, implying that ZNRF3 is also a negative regulator not only of WNT, but also BMP signaling. Consistently, our results indicate that loss of ZNRF3 increases BMP signaling. Moreover, ZNRF3 overexpression induces expression of Spemann organizer genes in Xenopus embryos, which is characteristic not only for WNT but also BMP inhibition ${ }^{43}$. In WNT signaling, the role of RSPO2 is to protect WNT receptors from ubiquitination and internalization by ZNRF3, by forming a ternary complex with LGR4-6 and triggering endocytosis. In contrast, during BMP signaling, RSPO2 directly forms a ternary complex with ZNRF3-BMPR1A to internalize and degrade the type I receptor. Our data also imply a possible function of RNF43 in antagonizing BMP signaling, inviting a closer inspection of its loss-of-function phenotypes 25,26 .

A number of studies emphasized the importance of the Furin domains in RSPOs, which are necessary and sufficient for activation of WNT signaling ${ }^{17,20,26,28}$, however, the role of the TSP1 domain has received less attention. We found that the specificity of RSPOs for BMP signaling is dictated by the TSP1 domain, which binds directly to BMPR1A. Unlike RSPO2 and RSPO3, RSPO1 and RSPO4 do not inhibit BMP signaling, the key difference residing in the TSP1 domain, as domain swapping of the TSP1 domain is sufficient to confer BMP inhibition upon RSPO1. 
a

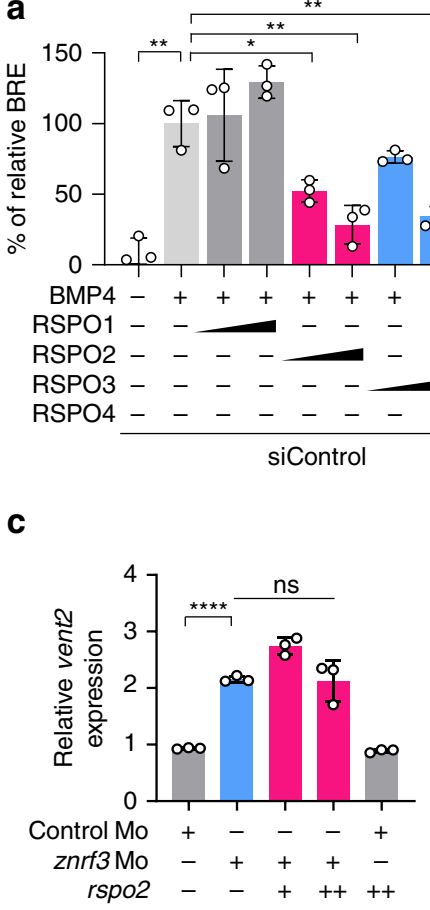

ns

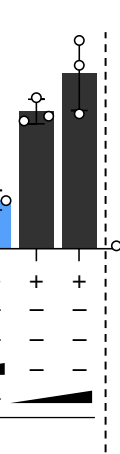

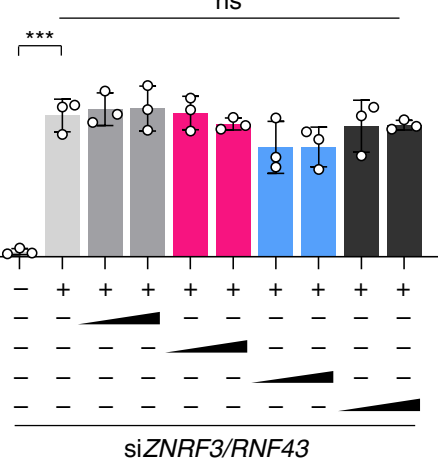

d

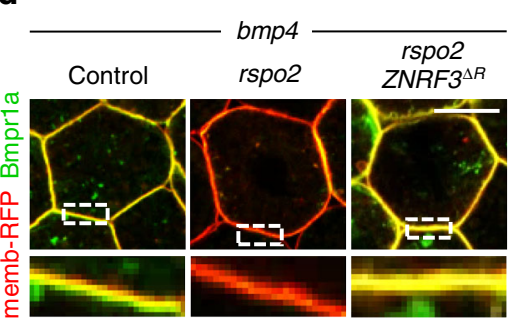

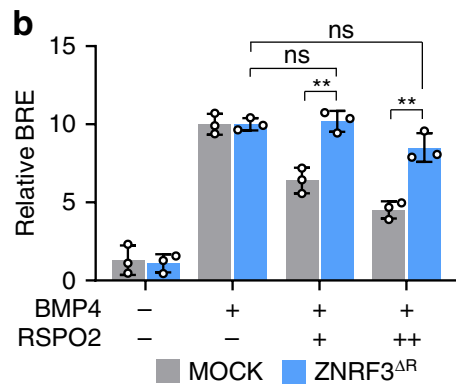
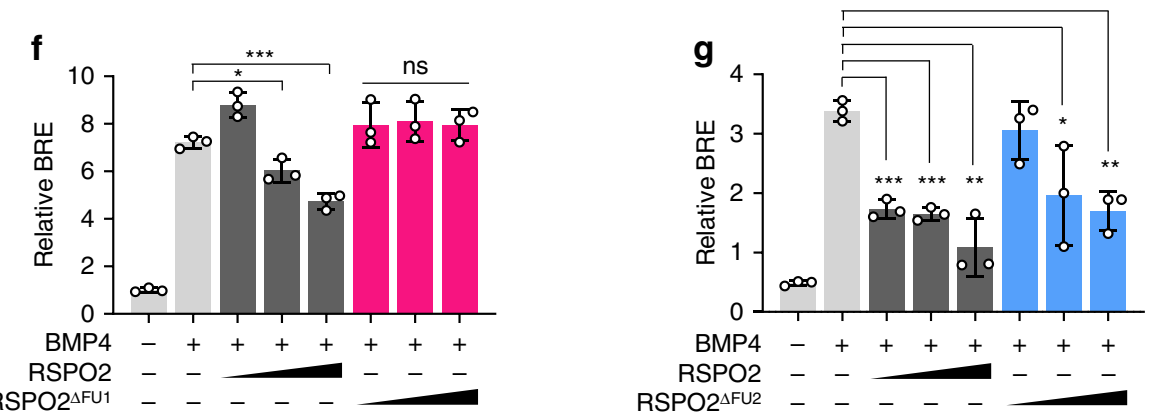

h

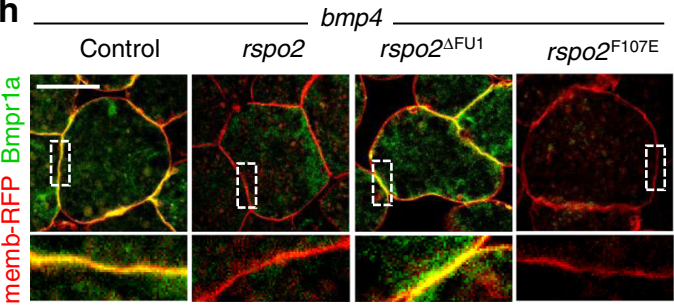

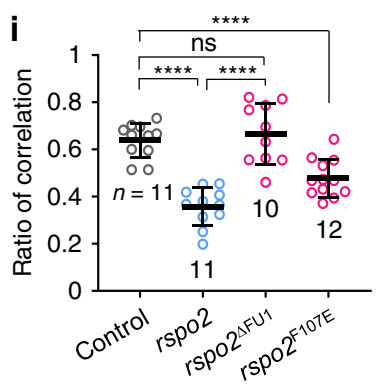

Fig. 7 RSPO2 requires ZNRF3 to antagonize BMP4-BMPR1A signaling. a BRE reporter assay in HEPG2 cells. Cells were transfected with siControl or siZNRF3/siRNF43, and BMP4 with or without RSPO1-4 were added overnight as indicated. Normalized BRE activity upon BMP4 without RSPO2 stimulation was set to $100 \% . n=3$ biologically independent samples. b BRE reporter assay in HEPG2 cells upon ZNRF3 $\triangle R$ transfection, with or without overnight BMP4 and RSPO2 treatment as indicated. $n=3$ biologically independent samples. c BMP-reporter (vent2) assay in Xenopus laevis St.15 neurulae. Embryos were injected animally with reporter plasmids and the indicated Mo with or without rspo2 mRNA at 4-cell stage. Normalized vent2 activity of control Mo injected embryos with reporter plasmids was set to $1 . n=3$ biologically independent samples. $\mathbf{d}$ Immunofluorescence microscopy (IF) in Xenopus laevis animal cap explants for Bmprla (green) and the plasma membrane (red) from embryos injected with mRNA as indicated, with a representative cell (top) and magnification (inset). Scale bar, $20 \mu \mathrm{m}$. For scheme, see Fig. 6 c. e Quantification of $\mathbf{d}$. $n=$ areas analyzed and data are displayed as mean \pm SD. ns, not significant, ${ }^{\star \star \star \star} P<0.0001$ from one-way ANOVA analysis. $\mathbf{f}-\mathbf{g}$ BRE reporter assay in HEPG2 cells treated with BMP4 and RSPO2/RSPO2 ${ }^{\triangle F U 1} / R_{S P P O}{ }^{\Delta F U 2}$ overnight as indicated. For domain structure of RSPO2 ${ }^{\triangle F U 1} / R_{S P O} \triangle \mathrm{FU} 2$, see Supplementary Fig. $8 \mathrm{a} . n=3$ biologically independent samples. $\mathbf{h}$ IF for Bmpr1a (green) and plasma membrane (red) in animal cap explants injected as indicated, with a representative cell (top) and magnification (inset) showing the plasma membrane. Scale bar, $20 \mu \mathrm{m}$. For domain structure of Xenopus Rspo2 mutants, see Supplementary Fig. 8d. $\mathbf{i}$ Quantification of $\mathbf{h} . n=$ areas analyzed and data are displayed as mean \pm SD. ns, not significant, ${ }^{\star \star \star \star} P<0.0001$ from one-way ANOVA analysis. All data are displayed as mean \pm SD. The $P$ values for reporter assays $\left(\mathbf{a}-\mathbf{c}, \mathbf{f}, \mathbf{g}\right.$ ) were determined from two-tailed unpaired $t$-test. ns, not significant; ${ }^{\star} P<0.05,{ }^{\star \star} P<0.01,{ }^{\star \star \star} P<0.001,{ }^{\star \star \star \star} P<0.0001$. 

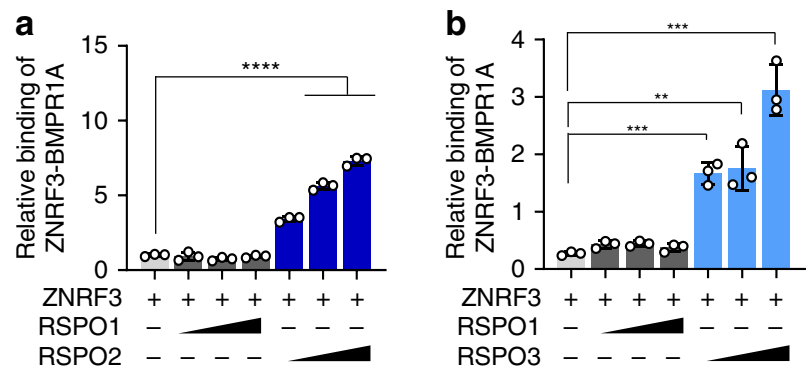

BMPR1A-HA ZNRF3-Flag Nucleus
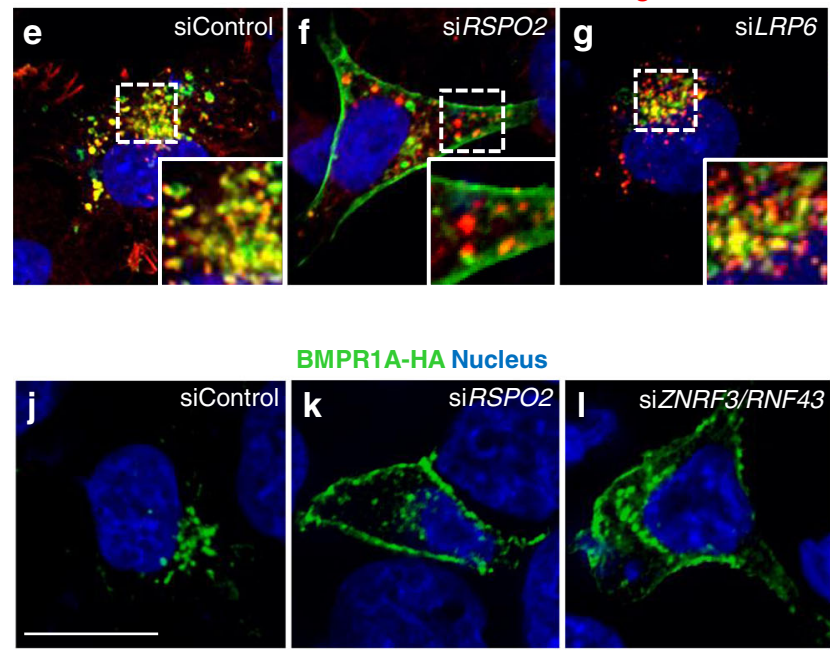

BMPR1A-HA Nucleus
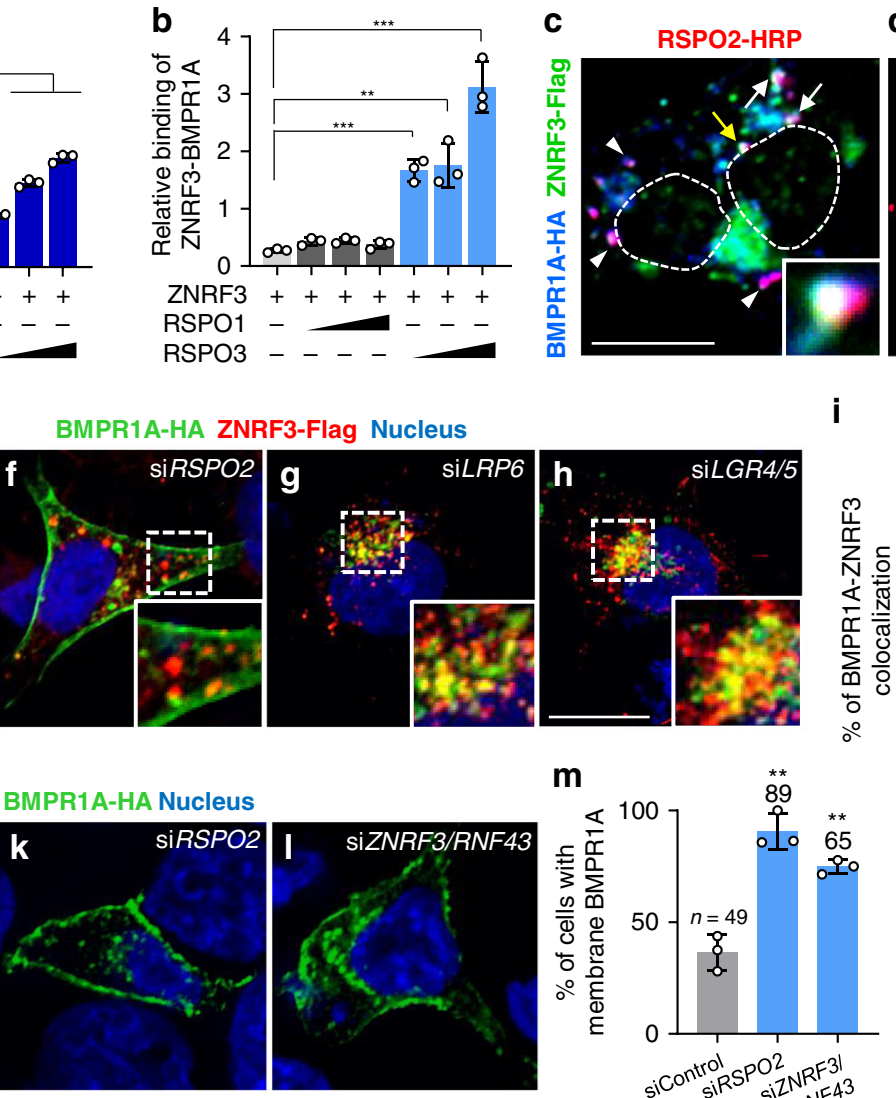

d

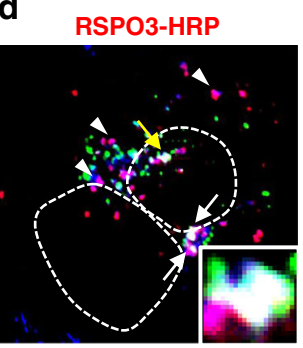

i

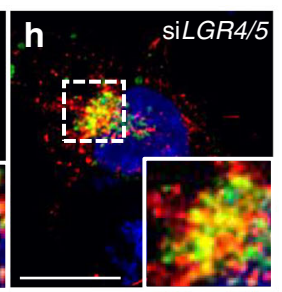

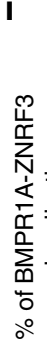

m
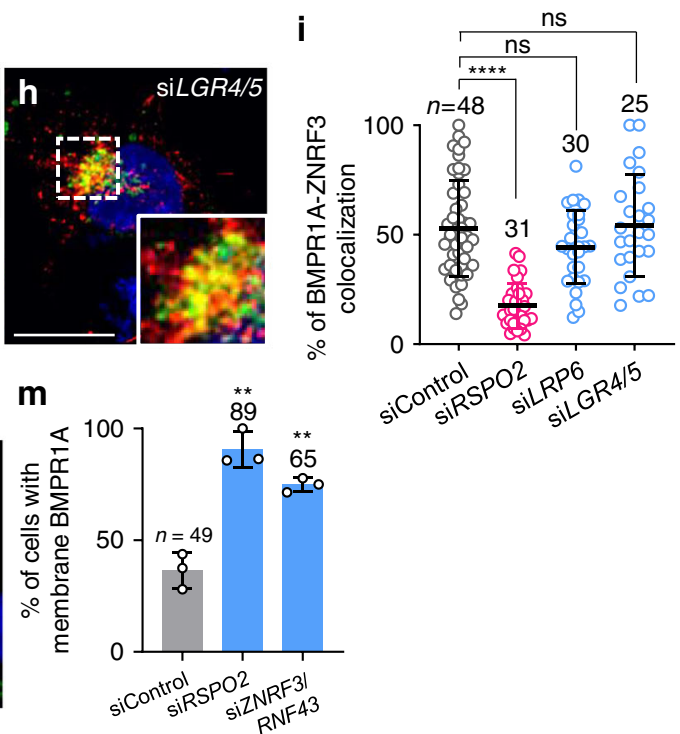

n

Cell surface biotinylation
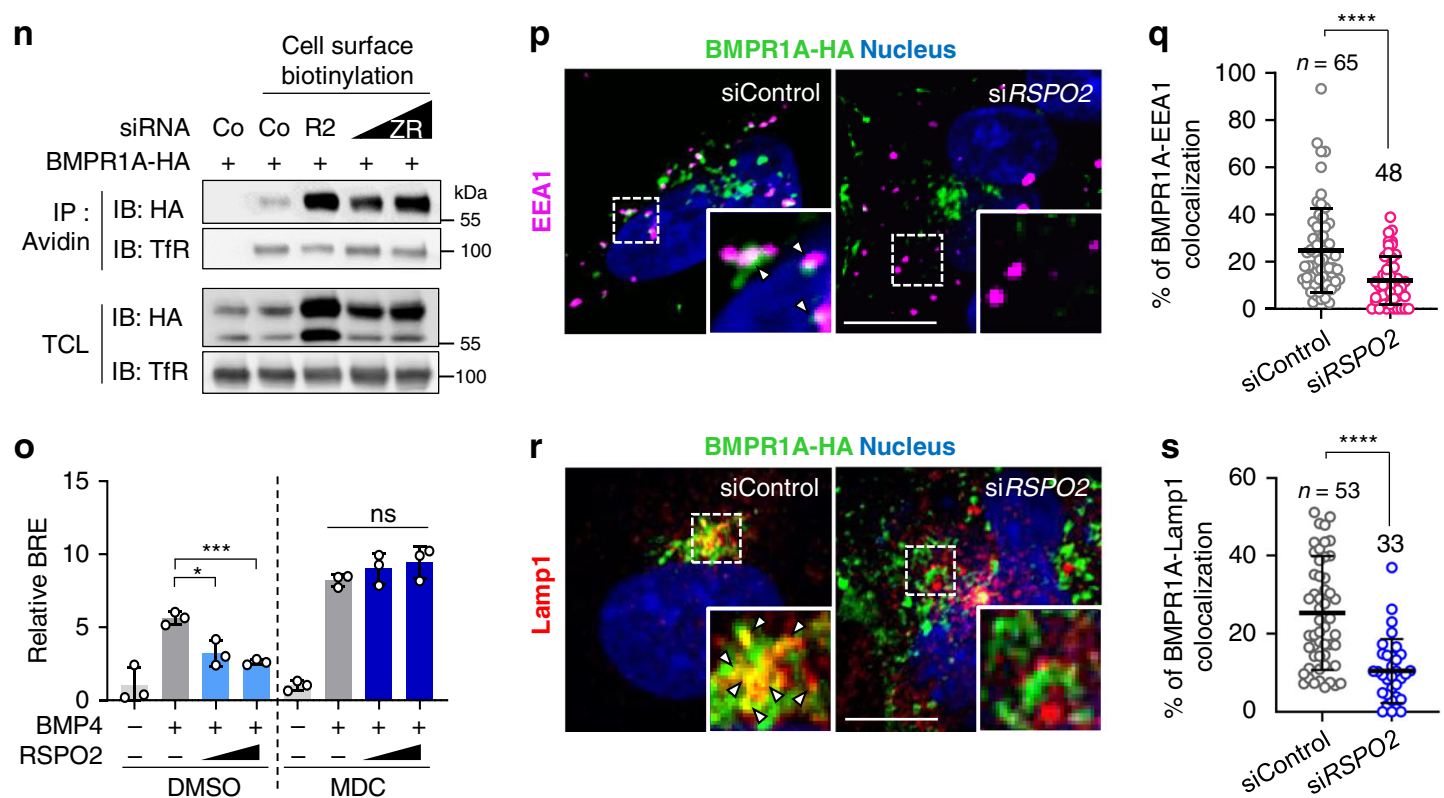

The physiological role in vivo is highlighted by rspo2 Morphants specifically lacking the TSP1 domain, which displayed phenotypic defects due to BMP hyperactivation (Fig. 5). The TSP1 domain also binds to heparin sulfate proteoglycans (HSPG) e.g., syndecans $(\mathrm{SDC})^{27,29,30}$, which raises the possibility of cooperation between RSPOs and SDC in BMP receptor regulation. Indeed, SDC1 and SDC3 have been implicated as negative regulators in BMP signaling, but the underlying mechanisms remained unclear $^{47,48}$. Hence, it will be interesting to investigate the role of SDCs in BMPR1A-RSPO interactions. HSPGs are also coreceptors in FGF signaling, which may explain why misexpressed rspo 2 can inhibit FGF signaling in Xenopus animal cap explants ${ }^{49}$.

We established that Xenopus Rspo2 cooperates with Noggin and Chordin released by the Spemann organizer in repressing BMP signaling to modulate the BMP morphogen gradient, which controls axial patterning. Yet, overexpression of $r s p o 2$ unlike of noggin and chordin, does not strongly dorsalize early embryos. The reason is that instead of sequestering BMP ligands, RSPO2 specifically targets the BMP receptor BMPR1A in early 
Fig. 8 RSPO2 bridges BMPR1A and ZNRF3 and triggers BMP receptor clearance from the cell surface. $\mathbf{a}$, $\mathbf{b}$ In vitro binding assay between ZNRF3 and BMPR1AECD mediated by RSPO1-3. ZNRF3-Fc protein was used as a bait, with sequential RSPO1-3 protein and BMPR1AECD-AP treatment. Bound BMPR1AECD to ZNRF3 was detected by chromogenic AP assay. $n=3$ experimentally independent samples. $\mathbf{c}$, $\mathbf{d}$ IF in H1581 cells transfected with BMPR1AHA and ZNRF3-flag DNA upon RSPO2 and RSPO3-HRP treatment for $3 \mathrm{~h}$. RSPOs (red) were visualized with tyramid signal amplification. BMPR1A (blue) and ZNRF3 (green) were stained against HA and flag antibody. White arrowheads, colocalized BMPR1A/RSPO2; white arrows, colocalized BMPR1A/ RSPO2-3/ZNRF3; yellow arrow, colocalized BMPR1A/RSPO2-3/ZNRF3 in magnified inset; Dashed lines, nucleus. Scale bar, $20 \mu \mathrm{m}$. e-h IF of colocalized BMPR1A (green)/ZNRF3 (red) in H1581 cells treated with siRNA as indicated. Nuclei were stained with Hoechst. Scale bar, $20 \mu \mathrm{m}$. i Quantification of BMPR1A colocalizing with ZNRF3 from e-h. j-I IF of BMPR1A (green) in H1581 cells treated with siRNA as indicated. $\mathbf{m}$ Quantification of cells harboring membrane localized BMPR1A from $\mathbf{j}-\mathbf{l}$. Scale bar, $20 \mu \mathrm{m}$. $\mathbf{n}$ Cell surface biotinylation assay in H1581 cells treated with BMPR1A-HA and siRNA as indicated. Co, control; R2, RSPO2; ZR, ZNRF3/RNF43 siRNA. After labeling surface proteins with Biotin, lysates were pulled down with streptavidin beads and subjected to Western blot analysis. Transferrin receptor (TfR), a loading control. TCL, Total cell lysate. Data shows results representative for 2 from 3 independent experiments. o BRE reporter assay in HEPG2 cells treated as indicated. MDC, monodansylcadaverin. $n=3$ biological replicates. $\mathbf{p}$ IF of colocalized BMPR1A (green)/EEA1 (magenta) in H1581 cells treated with siRNA. White arrowheads, colocalized BMPR1A/EEA1 in magnified inset. q Quantification of $\mathbf{p}$. Scale bar, $20 \mu \mathrm{m}$. $\mathbf{r}$ IF of colocalized BMPR1A (green)/Lamp1 (red) in H1581 cells treated with siRNA as indicated. White arrowheads, colocalized BMPR1A/Lamp1 in magnified inset. Scale bar, $20 \mu \mathrm{m}$. $\mathbf{s}$ Quantification of $\mathbf{r}$. For $\mathbf{i}, \mathbf{m}, \mathbf{q}, \mathbf{s}, n=$ the number of cells pooled from 2 independent experiments. Data for $(\mathbf{a}, \mathbf{b}, \mathbf{i}, \mathbf{m}, \mathbf{0}, \mathbf{q}, \mathbf{s})$ are displayed as mean \pm SD. ns, not significant, ${ }^{\star} P<0.05,{ }^{\star \star} P<0.01,{ }^{\star \star \star} P<0.001,{ }^{\star \star \star \star} P<0.0001$ from two-tailed unpaired $t$-test.

Xenopus embryos and that BMPR1A and BMPR1B play overlapping roles in dorsoventral patterning ${ }^{50,51}$. Also unlike noggin and chordin, rspo2 is not expressed in the organizer but is a negative feedback inhibitor of the BMP4 synexpression group, similar to the BMP pseudoreceptor $b a m b i^{44,52}$. Like bambi, rspo2 is an indirect BMP4 target gene, which may require Vent or Msx transcription factors for expression. Negative feedback in BMP signaling expands the dynamic BMP signaling range essential for proper embryonic patterning and reduce inter-individual phenotypic and molecular variability in Xenopus embryos ${ }^{42}$. Indeed, rspo2 deficiency by itself has only mild effects on axis formation and dorsoventral marker gene expression, while defects manifest upon misbalance of BMP signaling (bmp4-overexpression, noggin/chordin knockdown). Functional redundancy between BMP antagonists is a characteristic feature observed in fish, frog, and mouse embryos ${ }^{53-56}$. We note that the mouse Rspo2 expression pattern at E9.5 mimics that of mouse Bmp4, including forebrain, midbrain/hindbrain junction, branchial arches and limb apical ectodermal ridge ${ }^{57,58}$. Thus, although Rspo2 deficient mouse embryos gastrulate normally ${ }^{59}$, it may be fruitful to analyze compound mutants between Rspo2 and BMP antagonists for axial defects. The fact that R-spondins are bifunctional ligands, which activate WNT signaling and inhibit BMP signaling has implications for development, stem cell biology, and cancer. Mechanistically, the general picture emerging is that R-spondins function as adapters, which escort client extracellular proteins for ZNRF3/RNF43-mediated degradation, e.g., LGR4-6 and BMPR1A. Our results assign a key role to the largely ignored TSP1 domain of R-spondins in providing target specificity. The substantial sequence variability between TSP1 domains of RSPO1-4 invites screening for additional RSPO receptor targets beyond BMPR1A.

\footnotetext{
Methods

Cell lines and growth conditions. HEK293T and HEPG2 cells (ATCC) were maintained in DMEM High glucose (Gibco 11960) supplemented with 10\% FBS (Capricorn FBS-12A), 1\% penicillin-streptomycin (Sigma P0781), and $2 \mathrm{mM} \mathrm{L-}$ glutamine (Sigma G7513). H1581 cells (gift from Dr. R. Thomas) were maintained in RPMI (Gibco 21875) with 10\% FBS, $1 \%$ penicillin-streptomycin, $2 \mathrm{mM} \mathrm{L-}$ glutamine and $1 \mathrm{mM}$ sodium pyruvate (Sigma S8636). Mycoplasma contamination was negative in all cell lines used.
}

Xenopus laevis and Xenopus tropicalis. Xenopus laevis frogs were obtained from Nasco. Xenopus tropicalis frogs were obtained from Nasco, National Xenopus Resource (NXR) and European Xenopus Resource Centre (EXRC).

All X.laevis and X.tropicalis experiments were approved by the state review board of Baden-Württemberg, Germany (permit number 35-9185.81/G-141/18 (Regierungspräsidium Karlsruhe)) and executed according to federal and institutional guidelines and regulations. Developmental stages of the embryos were determined according to Nieuwkoop and Faber (Xenbase). No statistical analysis was done to adjust sample size before the experiments. No randomization of injection order was used during the experiments.

Constructs. Alkaline phosphatase (AP) fusions with RSPOs (human RSPO1 $\triangle \mathrm{C}_{-}$ AP-pCDNA3, RSPO2 ${ }^{\triangle \mathrm{C}}$-AP-pCDNA3, RSPO2 ${ }^{\Delta \mathrm{C}}$-AP-pCS2+, RSPO $3^{\Delta \mathrm{C}}$-APpCDNA3, murine RSPO4 ${ }^{\Delta \mathrm{C}}$-pCDNA3) were generated by replacing the Cterminal domain $(\triangle \mathrm{C})$ by $\mathrm{AP}$ and used to produce conditioned media. Human RSPO2 wild-type (RSPO2), the Furin1 and the Furin2 domain deletion mutants $\left(\mathrm{RSPO} 2^{\triangle \mathrm{FU} 1 / 2}\right)$, and the TSP1 domain deletion mutant (RSPO2 $\left.{ }^{\Delta \mathrm{TSP}}\right)$ are ORFs lacking the C-terminal domain, C-terminally tagged with a Flag-tag and subcloned into pCS2 $+{ }^{20}$. R1-TSPR2, R1-TSP ${ }^{\mathrm{R} 2}-\mathrm{AP}$ and R1-TSP ${ }^{\mathrm{R} 2}-$ Flag plasmids were cloned in pCS2 2 . Human RSPO2 ${ }^{\triangle \mathrm{FU} 1}$ (deletion of amino acids encompassing the 6 cystines in the FU1 domain) and $\mathrm{RSPO} 2^{\Delta \mathrm{FU} 2}$ mutants (deletion of amino acids encompassing the 8 cystines in the FU2 domain) were cloned in Flag-tag or AP-tag pCS2+. Human RSPO1 ${ }^{\text {TSP1 }}$ and RSPO2 ${ }^{\text {TSP1 }}-$ HA were cloned in Streptag-HA-flagpCS2+. Xenopus Rspo $2^{\Delta \mathrm{FU} 1}$ (deletion of amino acids encompassing the 6 cystines in the FU1 domain) and Rspo2 ${ }^{\mathrm{F} 107 \mathrm{E}}$ mutants were cloned in Myc-tag or AP-tag pCS2+. All constructs were confirmed by sequencing. Conditioned media from all RSPO constructs were adjusted to equal concentration by western blot and AP activity measurement, and further validated by WNT reporter assay using HEK293T cells. The extracellular domain of BMPR1A (BMPR1A ${ }^{\mathrm{ECD}}$ ) was subcloned in $\mathrm{AP}-\mathrm{pCS} 2+$ for generating conditioned medium and used in in vitro binding assays. Constitutively active forms of ACVR1, BMPR1A, and BMPR1B (QD) were generated by Gln-Asp mutations as described ${ }^{60}$. HA-tagged BMPR1A/ ALK3 was a gift from Dr. D. Koinuma ${ }^{61}$.

For Xenopus mRNA microinjection, Xenopus laevis Bmp4-pCS2+, Rspo2 ${ }^{\Delta \mathrm{C}}$ myc-pCS2+, Rspo $2^{\Delta \mathrm{FU} 1 / 2}$-myc-pCS2+ and Rspo $2^{\Delta \mathrm{TSP}}{ }_{-m y c-p C S 2+}$ plasmids, Bmprla ${ }^{\mathrm{DN}}-\mathrm{pCS} 2+$, membrane-RFP, Bmprla-EYFP-pCS2+, Rspo2 ${ }^{\Delta \mathrm{FU} 1}$-mycpCS2+, Rspo2 $2^{\mathrm{F} 107 \mathrm{E}}-$ myc-pCS2+ were used for in vitro transcription. Human Noggin-AP-pCS2+ and Chordin-AP-pCS2+ plasmids were used for Xenopus tropicalis Crispant rescue assay. Human ZNRF3 and ZNRF $3^{\Delta R I N G}$ constructs were gifts from Dr. F. Cong (Novartis) ${ }^{26}$, and ORFs were further subcloned in flag-pCS2+ for in vitro transcription.

Cell transfection. For HEPG2 and H1581 cells, siRNAs and plasmids were transfected using DharmaFECT 1 transfection reagent (Dharmacon T-2001) and Lipofectamine 3000 (Invitrogen L3000), respectively, according to the manufacturer protocols. For HEK293T cells, X-tremeGENE 9 DNA transfection reagent (Roche 6365787001) was used, according to the manufacturer protocols.

Generation of conditioned medium. HEK293T cells were seeded in $15 \mathrm{~cm}$ culture dishes and transiently transfected with RSPOs-AP, RSPOs-flag, BMPR1A ${ }^{\mathrm{ECD}}$-AP, DKK1 or WNT surrogate plasmids. After $24 \mathrm{~h}$, media were changed with fresh DMEM, 10\% FBS, $1 \%$ L-glutamine and $1 \%$ penicillin-streptomycin and cultured 6 days at $32^{\circ} \mathrm{C}$. Conditioned media were harvested three times every two days, centrifuged and validated by TOPFlash assay or western blot analyses. Mouse WNT3A conditioned medium was produced from mouse L-cells stably transfected with WNT3A (ATCC CRL-2647) following the manufacturer's instruction. For human $\mathrm{RSPO} 2^{\Delta \mathrm{FU} 1}, \mathrm{RSPO} 2^{\Delta \mathrm{FU} 2}$, Xenopus $\mathrm{Rspo}{ }^{\Delta \mathrm{FU}}$, and $\mathrm{Rspo} 2^{\mathrm{F} 107 \mathrm{E}}$ mutants conditioned media, HEK293T cells were seeded in 12 well culture plates and transfected with $500 \mu \mathrm{g}$ of each plasmid, and harvested three times every two days. Production of the media was validated with western blot analyses and AP activity analyses. 
Luciferase reporter assays. BRE luciferase assays were executed using $300,000 \mathrm{ml}^{-1}$ of HEPG2 cells in 24-well plates. PGL3-BRE-Luciferase $\left(500 \mathrm{ng} \mathrm{ml}^{-1}\right)$ and pRL-TKRenilla plasmids ( $50 \mathrm{ng} \mathrm{ml}^{-1}$ ) were transfected using Lipofectamine 3000 . After $24 \mathrm{~h}$, cells were serum starved $2 \mathrm{~h}$ and stimulated $14-16 \mathrm{~h}$ with $80 \mathrm{ng} \mathrm{ml}^{-1}$ recombinant human BMP4 protein (R\&D systems 314-BP) along with RSPO1-4 conditioned medium. TOPFlash luciferase assays were executed in HEK293T cells using 10,000 cells per well in 96-well plates. TOPFlash-Luciferase $(5 \mathrm{ng})$ and pRL-TK-Renilla plasmids (1 ng) per well were transfected using X-tremeGENE9 transfection reagent (Roche 06365787001 ). After $48 \mathrm{~h}$, cells were stimulated $24 \mathrm{~h}$ with WNT3A conditioned medium along with RSPO conditioned medium. Luciferase activity was measured with the Dual luciferase reporter assay system (Promega E1960). Firefly luminescence (BRE or TOPFlash) was normalized to Renilla. Statistical analyses were made with Graphpad PRISM7 software.

Western blot analysis. Cultured cells were rinsed with cold PBS and lysed in Triton lysis buffer (20 mN Tris-Cl, pH 7.5, 1\% Triton X-100, $150 \mathrm{mM} \mathrm{NaCl}, 1 \mathrm{mM}$ EDTA, $1 \mathrm{mM}$ EGTA, $1 \mathrm{mM} \beta$-glycerophosphate, $1 \mathrm{mM} \mathrm{Na}_{3} \mathrm{VO}_{4}$ ) or RIPA buffer with cOmplete Protease Inhibitor Cocktail (Roche 11697498001). Lysates were mixed with Laemmli buffer containing $\beta$-mercaptoethanol and boiled at $95^{\circ} \mathrm{C}$ for $5 \mathrm{~min}$ to prepare SDS-PAGE samples. Western blot images were acquired with SuperSignal West pico ECL (ThermoFisher 34580) or Clarity Western ECL (Biorad 1705061) using LAS-3000 system (FujiFilm). Quantification of blots was done using Image J v1.51k software.

Cell surface biotinylation assay. H1581 cells were seeded in $6 \mathrm{~cm}$ culture dishes and transfected with $50 \mathrm{nM}$ of indicated siRNAs for 3 days and $2 \mu \mathrm{g}$ of BMPR1AHA DNA for 2 days. Surface proteins were biotinylated with $0.25 \mathrm{mg} \mathrm{ml}^{-1}$ sulfoNHS-LC-LC-Biotin (ThermoFisher 21338) at $4{ }^{\circ} \mathrm{C}$ for $30 \mathrm{~min}$. The reaction was quenched by $10 \mathrm{mM}$ Monoethanolamine and cells were harvested and lysed with Triton X-100 lysis buffer. 200-300 $\mu$ g of lysate was incubated with $20 \mu \mathrm{l}$ streptavidin agarose (ThermoFisher 20359) to pull-down biotinylated surface proteins and subjected to Western blot.

Surface receptor internalization assay. $\mathrm{H} 1581$ cells were seeded in $15 \mathrm{~cm}$ culture dish and transfected with $10 \mu \mathrm{g}$ of BMPR1A-HA DNA for 2 days, and then split to $6 \mathrm{~cm}$ culture dishes. After $24 \mathrm{~h}$, surface proteins were biotinylated with $0.5 \mathrm{mg} \mathrm{ml}^{-1}$ sulfo-NHS-SS-Biotin (ThermoFisher 21331) at $4{ }^{\circ} \mathrm{C}$ for $30 \mathrm{~min}$. After quenching excessive biotin with $10 \mathrm{mM}$ monoethanolamine, pre-warmed control medium or RSPO2 conditioned medium was added at $37^{\circ} \mathrm{C}$ to induce internalization. After 20 min stimulation, remaining surface-biotin was removed by $50 \mathrm{mM}$ MesNa (2mercaptoethanesulfonate, membrane impermeable reducing agent, CAYMAN $21238)$ in MesNa reaction buffer ( $100 \mathrm{mM}$ Tris- $\mathrm{HCl}, \mathrm{pH} 8.6,100 \mathrm{mM} \mathrm{NaCl}$ and $2.5 \mathrm{mM} \mathrm{CaCl}_{2}$ ) at $4{ }^{\circ} \mathrm{C}$ for $30 \mathrm{~min}$ and MesNa protected-biotinylated proteins (internalized proteins) were analyzed. Cells were harvested, and lysed with RIPA buffer $(20 \mathrm{mM}$ Tris-Cl, pH 7.4, $120 \mathrm{mM} \mathrm{NaCl}, 1 \%$ Triton X-100, 0.25\% Nadeoxycholate, $0.05 \%$ SDS, $50 \mathrm{mM}$ sodium fluoride, $5 \mathrm{mM}$ EDTA, $2 \mathrm{mM} \mathrm{Na}$ orthovanadate) supplemented with complete protease inhibitor. Five hundred microgram of lysate was incubated with $20 \mu \mathrm{l}$ streptavidin agarose (ThermoFisher 20359) to pull-down biotinylated proteins and subjected to Western blot.

Xenopus laevis whole-mount in situ hybridization. Whole-mount in situ hybridizations of Xenopus embryos were performed using digoxigenin (DIG)-labeled probes according to the standard protocol (https://www.xenbase.org) ${ }^{62}$. Antisense RNA probes against $r s p o 2$ and $b m p 4$ were generated by in vitro transcription with T7 RNA polymerase (Promega P2075) ${ }^{20}$. Probes against bmprla and znrf3 were prepared using full-size Xenopus bmprla ORF or znrf3 ORF as a template, linearized with Xho I (NEB R0146S) and transcribed with T7 RNA polymerase. Mo and mRNA injected embryos were collected at stage 11 (gastrula) or 32 (tadpole) for in situ hybridization. Images were obtained using AxioCam MRc 5 microscope (Zeiss). Embryos in each image were selected using Magnetic Lasso tool or Magic Wand tool of Adobe Photoshop CS6 software, and pasted into the uniform background color for presentation.

Xenopus microinjection and phenotype analysis. In vitro fertilization, microinjection and culture of Xenopus embryos were performed according to the standard protocol (https://www.xenbase.org). X.laevis embryos were microinjected with reporter DNAs, in vitro transcribed mRNAs or antisense morpholino oligonucleotide (Mo) using Harvard Apparatus microinjection system. Mos for $r s p o 2^{20}$, $\operatorname{lrp} 6^{39}$, chordin, bmp $4^{38}, z n r f 3^{43}$ and standard control were purchased from GeneTools. $r s p o 2^{\Delta \mathrm{TSP}}$ Mo was designed based on $r s p o 2$ sequence (Supplementary Table 3). X.laevis 4-cell stage embryos were microinjected $5 \mathrm{nl}$ per each blastomere equatorially and cultured until indicated stages. Equal amount of total mRNA or Mo were injected by adjustment with $p p l$ or standard control Mo. Scoring of phenotypes was executed blind from two individuals, and data are representative images from at least two independent experiments. Embryos in each image were selected using Magnetic Lasso tool or Magic Wand tool of Adobe Photoshop CS6 software, and pasted into the uniform background color for presentation.
Xenopus tropicalis CRISPR/Cas9-mediated mutagenesis. The 5' region of genomic sequences from X.tropicalis chordin (NM_001142657.1) and noggin (NM_001171898.1) were searched for guide RNA (gRNA) targeting sites using an online prediction tool (https://crispr.cos.uni-heidelberg.de). Primers for $r s p o 2$, noggin and chordin were designed or chosen ${ }^{27}$ for PCR-based gRNA template assembly (Supplementary Table 4$)^{63}$. A primer lacking any target sequences was used as control gRNA. PCR reactions were performed with Phusion Hot Start Flex DNA Polymerase (NEB M0535), followed by in vitro transcription using MEGAscript T7 Transcription Kit (Invitrogen AM1334). Embryos were microinjected at one to two-cell stages with a mixture of $50 \mathrm{pg}$ of gRNA and $1 \mathrm{ng}$ of recombinant Cas9 protein (Toolgen) per embryo. Injected embryos were cultured until stage 30, fixed with MEMFA for phenotypical analysis. Scoring of phenotypes was executed at stage 30 with blinding from two individuals, and data are representative images from three independent experiments. Defects were categorized by the severity of ventralization. 'Severe' showed small head, enlarged ventral tissues and short body axis. 'Mild' showed one or two of the defects described above. 'Normal' showed no visible differences to the uninjected control.

Injected amount of reagents per Xenopus embryo. Equal amounts of total RNA or Mo were injected by adjustment with preprolactin (PPL) mRNA, control gRNA or standard control Mo. Per embryo; Fig. 2b, $250 \mathrm{pg}$ of $b m p 4$, rspo2 and $r s p o 2$ mutants mRNA; Fig. $2 \mathrm{c}, 5 \mathrm{ng}$ or $10 \mathrm{ng}$ of $r s p o 2 \mathrm{Mo}$ and $5 \mathrm{ng}$ of $l$ rp $6 \mathrm{Mo}, 300 \mathrm{pg}$ of reporter DNA; Fig. 2d, $15 \mathrm{ng}$ of $b m p 4 \mathrm{Mo}, 2,5$, or $10 \mathrm{ng}$ of $r s p o 2 \mathrm{Mo}, 300 \mathrm{pg}$ of reporter DNA; Fig. $2 \mathrm{f}, 15 \mathrm{ng}$ of bmp4 Mo and $5 \mathrm{ng}$ of $r s p o 2 \mathrm{Mo}$; Fig. $2 \mathrm{~h}, 50 \mathrm{pg}$ of gRNA, $200 \mathrm{pg}$ of $b m p 4$ mRNA, 2 ng of lrp6 Mo, $1 \mathrm{ng}$ of Cas9 protein; Fig. 3c, e, 500 pg of $b m p 4$; Fig. $5 \mathrm{~b}, \mathrm{c}, 20 \mathrm{ng}$ of $r s p o 2 \mathrm{Mo}$ and $r s p o 2^{\Delta \mathrm{TSP}} \mathrm{Mo}, 300 \mathrm{pg}$ of reporter DNA; Fig. 5e, g, $8 \mathrm{ng}$ of $c h d \mathrm{Mo}, 20 \mathrm{ng}$ of $r s p o 2^{\Delta \mathrm{TSP}} \mathrm{Mo}$ and $200 \mathrm{pg}$ of $b m p r 1 a^{\mathrm{DN}}$; Fig. $6 c, 500 \mathrm{pg}$ of bmp4 and bmpr1a-EYFP, $250 \mathrm{pg}$ of membrane-RFP, rspo2, and rspo 2 deletion mutants mRNA; Fig. $6 \mathrm{e}, 500 \mathrm{pg}$ of bmprla-EYFP, $250 \mathrm{pg}$ of $r s p o 2$, and $r s p o 2$ deletion mutants, and $g f p$; Fig. $6 \mathrm{~g}, \mathrm{i}, 10 \mathrm{ng}$ of $r s p o 2$ Mo and $r s p o 2^{\Delta T S P}$ Mo; Fig. $7 \mathrm{c}, 40 \mathrm{ng}$ of znrf3 Mo, $100 \mathrm{pg}$ and $200 \mathrm{pg}$ of $r s p o 2 \mathrm{mRNA}$, and $300 \mathrm{pg}$ of reporter DNA; Fig. 7d, $500 \mathrm{pg}$ of bmp4 and bmprla-EYFP, $250 \mathrm{pg}$ of membraneRFP, $250 \mathrm{pg}$ of $r s p o 2,100 \mathrm{pg}$ of $z n r f 3^{\mathrm{DN}}$ mRNA; Fig. 7h, $500 \mathrm{pg}$ of $b m p 4$ and bmprla-EYFP, $250 \mathrm{pg}$ of membrane-RFP, $r s p o 2$, and $r s p o 2$ mutants mRNA; Supplementary Fig. $2 \mathrm{~b}, 15 \mathrm{ng}$ of $b m p 4 \mathrm{Mo}$ and $5 \mathrm{ng}$ of $r s p o 2 \mathrm{Mo}$; Supplementary Fig. 3g, i, 50 pg of gRNA, $10 \mathrm{pg}$ and $25 \mathrm{pg}$ of chordin and noggin DNA; Supplementary Fig. 4b, d, f, 50 pg of gRNA, 200 pg of $b m p 4$ mRNA, 2 ng of $\operatorname{lrp} 6 \mathrm{Mo}, 1 \mathrm{ng}$ of Cas9 protein; Supplementary Fig. 4h, $250 \mathrm{pg}$ of $b m p 4$ and rspo3 mRNA; Supplementary Fig. $6 \mathrm{~b}, 20 \mathrm{ng}$ of $r s p o 2^{\Delta \mathrm{TSP}} \mathrm{Mo}, 150 \mathrm{ng}$ and $250 \mathrm{ng}$ of $r$ spo $2 \mathrm{mRNA}$ Supplementary Fig. $6 \mathrm{~d}, 15 \mathrm{ng}$ chd Mo, $10 \mathrm{ng}$ rspo2 Mo and $50 \mathrm{pg}$ bmprla ${ }^{\mathrm{DN}}$; Supplementary Fig. 7e, $80 \mathrm{ng}$ of znrf3 Mo, $200 \mathrm{pg}$ of ZNRF3 mRNA; Supplementary Fig. $10 c, 500$ pg of bmprla-EYFP.

Xenopus tropicalis T7 Endonuclease I assay. To validate CRISPR/Cas9-mediated genome editing, three embryos of each injection set were lysed at stage 30 for genotyping PCR reactions as described ${ }^{63}$. For primer sequences, see Supplementary Table 4 . All target sequences were amplified with Roti-Pol Hot-TaqS Mix (Roth 9248). After denaturation for $3 \mathrm{~min}$ at $94{ }^{\circ} \mathrm{C}$ and reannealing (ramp $0.1{ }^{\circ} \mathrm{C}$ per sec), the PCR products were incubated with $3 \mathrm{U}$ of T7 Endonuclease I for $45 \mathrm{~min}$ at $37^{\circ}$ C. Cleavage results were visualized on a $2 \%$ agarose gel.

Xenopus laevis western blot analysis. Injected Xenopus embryos were harvested at stage 15 to 18 , homogenized in NP- 40 lysis buffer ( $2 \% \mathrm{NP}-40,20 \mathrm{mM}$ Tris- $\mathrm{HCl}$ pH 7.5, $150 \mathrm{mM} \mathrm{NaCl}, 10 \mathrm{mM} \mathrm{NaF}, 10 \mathrm{mM} \mathrm{Na}_{3} \mathrm{VO}_{4}, 10 \mathrm{mM}$ sodium pyrophosphate, $5 \mathrm{mM}$ EDTA, $1 \mathrm{mM}$ EGTA, $1 \mathrm{mM}$ PMSF, and cOmplete Protease Inhibitor Cocktail) with a volume of $20 \mu \mathrm{l}$ per embryo. Lysates were cleared with CFC-113 (Honeywell 34874 ), followed by centrifugation $\left(18,800 \times g, 10 \mathrm{~min}\right.$ at $4{ }^{\circ} \mathrm{C}$ ), boiling at $95^{\circ} \mathrm{C}$ for $5 \mathrm{~min}$ with NuPAGE Sample Buffer. 0.5-1 embryos per lane were loaded for SDS-PAGE analysis.

Cycloheximide treatment on Xenopus laevis animal cap explants. Xenopus laevis animal caps were dissected at stage 8 and treated with $30 \mu \mathrm{g} \mathrm{ml}^{-1}$ of cycloheximide (CHX) (Sigma C7698) until control embryos reached stage 10. CHX treatment was validated since cell division was retarded compared to untreated control. In situ hybridization and qRT-PCR were performed with same methods used in whole embryos.

In vitro binding assay. High binding 96-well plates (Greiner M5811) were coated with $2 \mu \mathrm{g} \mathrm{ml}^{-1}$ of recombinant human RSPO1 (Peprotech 120-38), RSPO2 (Peprotech 120-43), RSPO3 (Peprotech 120-44), RSPO4 (R\&D systems 4575-RS) or FGF8b (Peprotech 100-25) recombinant protein reconstituted in bicarbonate coating buffer $\left(50 \mathrm{mM} \mathrm{NaHCO}_{3}, \mathrm{pH} 9.6\right)$ overnight at $4{ }^{\circ} \mathrm{C}$. Coated wells were washed three times with TBST (TBS, $0.1 \%$ Tween-20) and blocked with 5\% BSA in TBST for $1 \mathrm{~h}$ at room temperature. $1.5 \mathrm{U} \mathrm{ml}^{-1}$ of BMPR1A $\mathrm{ACD}_{-\mathrm{AP}}$ or control conditioned medium was incubated overnight at $4^{\circ} \mathrm{C}$. Wells were washed six times with TBST and bound AP activity was measured by the chemiluminescent SEAP Reporter Gene Assay kit (Abcam ab133077) or AquaSpark AP substrate (Serva 42593.01). For ZNRF3-BMPR1A binding assay, plates were coated with recombinant human ZNRF3 Fc Chimera protein (R\&D systems 7994-RF). RSPO2-flag, 
RSPO2 deletion mutants-flag conditioned medium, or recombinant RSPO protein was preincubated $4-6 \mathrm{~h}$ with $\mathrm{ZNRF} 3$ prior to $\mathrm{BMPR} 1 \mathrm{~A}^{\mathrm{ECD}}$ - $\mathrm{AP}$ treatment. Control conditioned medium and vesicles were used as control. Data show average chemiluminescent activities with SD from experimental triplicates. Statistical analyses show unpaired $t$-tests. The $K_{\mathrm{d}}$ was obtained with In vitro binding assay using RSPO2 recombinant protein (Peprotech 120-43) and BMPR1A ${ }^{\mathrm{ECD}}-\mathrm{AP}^{24}$.

Immunofluorescence. 150,000 H1581 cells were grown on coverslips in 12-well plates, followed by siRNA and DNA transfection. After $48 \mathrm{~h}$ cells were fixed in $4 \%$ PFA for $10 \mathrm{~min}$. Cells were treated with primary antibodies (1:250) overnight at $4{ }^{\circ} \mathrm{C}$, and secondary antibodies $(1: 500)$ and Hoechst dye $(1: 500)$ were applied for $2 \mathrm{~h}$ at room temperature. For Tyramide Signal Amplification to detect RSPOs, $\mathrm{H} 1581$ cells were treated with RSPO1-3 ${ }^{\Delta \mathrm{C}_{-}}$HRP conditioned media $3 \mathrm{~h}$ at $37^{\circ} \mathrm{C}$. Cells were washed with Hanks' balanced salt solution (HBSS) and fixed for $30 \mathrm{~min}$ with $0.5 \mathrm{mM}$ dithiobis (succinimidyl) propionate (DSP) (Thermo 22585) crosslinker in HBSS supplemented with $10 \mathrm{mM}$ HEPES, followed by permeabilization with $0.1 \%$ saponin in TSA buffer ( $100 \mathrm{mM}$ Tris, $\mathrm{pH} 8.8,10 \mathrm{mM}$ imidazole). The TSA reaction was executed for $30 \mathrm{~min}$ in dark with the $30 \mu \mathrm{M}$ tyramideRhodamine in TSA buffer supplemented with $0.003 \% \mathrm{H}_{2} \mathrm{O}_{2}$. Cells were washed and further stained with anti-HA and anti-Flag antibodies $(1: 250)^{24,39}$. Quantification was done using Image J v1.51k software.

For X. laevis embryos, bmprla-EYFP and membrane-RFP mRNAs were coinjected with the indicated mRNAs or Mos. Embryos were dissected for animal or ventrolateral explants at stage 9 or stage 11.5, respectively. Explants were immediately fixed with $4 \%$ PFA for $2 \mathrm{~h}$ and mounted with Fluoromount-G (ThermoFisher 00495802). Images were obtained using LSM 700 (Zeiss). Data are representative images from two independent experiments. For quantification, Pearson's correlation coefficient for EYFP and RFP was analyzed using 16-30 random areas harboring 10 cells chosen from 6-10 embryos per each set.

Cell surface binding assay. Two hundred and fifty nanogram of human BMPR1A-HA and Xenopus tropicalis LGR4 DNA were transfected in HEK293T cells and incubated with $1.5 \mathrm{U} \mathrm{ml}^{-1}$ conditioned media for $3 \mathrm{~h}$ on ice. After several washes with PBS and crosslinking with DSP, cells were treated with $2 \mathrm{mM}$ Levamisole for $20 \mathrm{~min}$ to inactivate endogenous AP activities and developed with BM-Purple (Sigma 11442074001). Cells were mounted with Fluoromount G. Images were obtained using LEICA DMIL microscope/Canon DS126311 camera.

Quantitative real-time PCR. Cultured cells were lysed in Macherey-Nagel RA1 buffer containing $1 \% \beta$-mercaptoethanol and total RNAs were isolated using NucleoSpin RNA isolation kit (Macherey-Nagel 740955). Reverse transcription and PCR amplification were performed as described before ${ }^{64}$. For Xenopus laevis, animal cap explants were harvested at stage 10 and lysed in $1 \mathrm{ml}$ of TRizol (Ambion 18914101). Extraction and precipitation of RNA were performed following the manufacturer's instruction. Reverse transcription was executed with 1 $\mu \mathrm{g}$ RNA using SuperScript II reverse transcriptase and random primers (Invitrogen $\mathrm{P} / \mathrm{N} 58875)$. PCR amplification with the obtained CDNA was performed using UPL (Universal Probe Library, Roche, \#15 (rspo2), \#140 (sizzled), \#145 (vent1)) probes and corresponding primers, further analyzed by LightCycle 480 . Primers used in this study are listed in Supplementary Table 1. Graphs show relative gene expressions to GAPDH. Data are displayed as mean with SD from multiple experimental replicates. Statistical analyses were performed using the GraphPad PRISM7 software.

Statistics and reproducibility. All exact $P$ values in the analyses are as follows. (Left to right of the graph) Fig. 1; (a), <0.0001, 0.0007, <0.0001, 0.0008; (b), $<0.0001,0.0007,0.0002,0.003,0.0013$; (e), 0.0064, 0.0009; (f), 0.0012, 0.0005; (h), $0.0006,<0.0001$; (j) $) 0.0347,0.0132,0.0040,0.1778,0.9918,0.0541,0.9925,0.9082$, $0.3433,0.9920,0.9991,0.8893$. Figure 2; (c), 0.0219, 0.1122, 0.0407, 0.0259; (d), $0.0153,0.0458,0.0233,0.0001,0.0096$; (h) $, 0.0007,0.0650,<0.0001,0.0035$. Figure 3 ; (c) $, 0.0039,0.0085,<0.0001,0.7836,0.0036,0.3596$. Figure 4 ; (a), 0.0013 , $0.0020,0.0007$; (b), 0.1193, 0.2732, 0.2591, 0.1096, 0.7246, 0.6867, 0.8089, 0.5742; (c), 0.9290, 0.9059, 0.1857, 0.0216, 0.4426, 0.9921, 0.9994, 0.9995; (e), 0.6542, $0.0007,0.0033,0.7650,0.2872 ;(\mathbf{h}), 0.0047 ;(\mathbf{j}), 0.0006,0.2410,0.8921 ;(\mathbf{k}), 0.0044$, $0.0005,0.0036$. Figure 5; (b), 0.0766, <0.0001; (c) $,<0.0001,0.0002$; (e), 0.0006, $<0.0001,0.0005$; (g) $,<0.0001,0.0019,<0.0001,<0.0001,<0.0001$. Figure 6; (e), $<0.0001,0.1072,0.0523$; (h), 0.0020, 0.0005. Figure 7; (a), 0.0011, 0.0104, 0.0043, $0.0031,0.0001,0.9979,0.9973,0.9997,0.9938,0.2378,0.2508,0.9775,0.9894 ;(\mathbf{b})$, $0.6982,0.0614,0.0034,0.0030$; (c) $<<0.0001,0.1144$; (e) $,<0.0001,0.5172,<0.0001$; (f), 0.0188, 0.0005, 0.8546, 0.9962; (g), 0.0001, 0.0003, 0.0016, 0.0465, 0.0014; (i), $<0.0001,0.5588,<0.0001,<0.0001$. Figure 8 ; (a) $,<0.0001,<0.0001,<0.0001$; (b), $0.0002,0.0026,0.0004 ;(\mathbf{i}),<0.0001,0.0643,0.8264 ;(\mathbf{m}), 0.0011,0.0015 ;(\mathbf{o}), 0.0137$, $0.0004,0.2874,0.1482 ;(\mathbf{q}),<0.0001 ;(\mathbf{s}),<0.0001$. For all $P$ values of Supplementary Figures, see Supplementary Information.
Reporting summary. Further information on research design is available in the Nature Research Reporting Summary linked to this article.

\section{Data availability}

NCBI Reference Sequences were used for Xenopus tropicalis chordin: [https://www.ncbi. nlm.nih.gov/nuccore/NM_001142657.1]; Xenopus tropicalis noggin: [https://www.ncbi. nlm.nih.gov/nuccore/NM_001171898.1]. All raw images of our blotting and all relevant data generated and analyzed in this study are provided in the Source Data file. Source data are provided with this paper.

Received: 12 March 2020; Accepted: 9 October 2020; Published online: 04 November 2020

\section{References}

1. David, C. J. \& Massague, J. Contextual determinants of TGFbeta action in development, immunity and cancer. Nat. Rev. Mol. Cell Biol. 19, 419-435 (2018).

2. Salazar, V. S., Gamer, L. W. \& Rosen, V. BMP signalling in skeletal development, disease and repair. Nat. Rev. Endocrinol. 12, 203-221 (2016).

3. Zhang, Y. \& Que, J. BMP signaling in development, stem cells, and diseases of the gastrointestinal tract. Annu. Rev. Physiol. 82, 251-273 (2020).

4. Zylbersztejn, F. et al. The BMP pathway: a unique tool to decode the origin and progression of leukemia. Exp. Hematol. 61, 36-44 (2018).

5. Wu, M., Chen, G. \& Li, Y. P. TGF-beta and BMP signaling in osteoblast, skeletal development, and bone formation, homeostasis and disease. Bone Res. 4, 16009 (2016).

6. Morrell, N. W. et al. Targeting BMP signalling in cardiovascular disease and anaemia. Nat. Rev. Cardiol. 13, 106-120 (2016).

7. Heldin, C. H. \& Moustakas, A. Signaling receptors for TGF-beta family members. Cold Spring Harb. Perspect. Biol. 8, a022053 (2016).

8. Antebi, Y. E. et al. Combinatorial signal perception in the BMP pathway. Cell 170, 1184-1196 e1124 (2017).

9. Massague, J. TGF-beta signal transduction. Annu. Rev. Biochem. 67, 753-791 (1998).

10. Heldin, C. H., Miyazono, K. \& ten Dijke, P. TGF-beta signalling from cell membrane to nucleus through SMAD proteins. Nature 390, 465-471 (1997).

11. Chang, C. Agonists and antagonists of TGF-beta family ligands. Cold Spring Harb. Perspect. Biol. 8, a021923 (2016).

12. Gamer, L. W., Nove, J., Levin, M. \& Rosen, V. BMP-3 is a novel inhibitor of both activin and BMP-4 signaling in Xenopus embryos. Dev. Biol. 285, 156-168 (2005).

13. Wiater, E. \& Vale, W. Inhibin is an antagonist of bone morphogenetic protein signaling. J. Biol. Chem. 278, 7934-7941 (2003).

14. Hao, H. X., Jiang, X. \& Cong, F. Control of Wnt receptor turnover by Rspondin-ZNRF3/RNF43 signaling module and its dysregulation in cancer. Cancers 8, 54 (2016).

15. Chartier, C. et al. Therapeutic targeting of tumor-derived R-spondin attenuates beta-catenin signaling and tumorigenesis in multiple cancer types. Cancer Res. 76, 713-723 (2016).

16. Seshagiri, S. et al. Recurrent R-spondin fusions in colon cancer. Nature 488 660-664 (2012)

17. de Lau, W., Peng, W. C., Gros, P. \& Clevers, H. The R-spondin/Lgr5/Rnf43 module: regulator of Wnt signal strength. Genes Dev. 28, 305-316 (2014).

18. Kim, K. A. et al. Mitogenic influence of human R-spondin1 on the intestinal epithelium. Science 309, 1256-1259 (2005).

19. Nanki, K. et al. Divergent routes toward Wnt and R-spondin niche independency during human gastric carcinogenesis. Cell 174, 856-869 e817 (2018).

20. Kazanskaya, O. et al. R-Spondin2 is a secreted activator of Wnt/beta-catenin signaling and is required for Xenopus myogenesis. Dev. Cell 7, 525-534 (2004).

21. Sato, T. et al. Single Lgr5 stem cells build crypt-villus structures in vitro without a mesenchymal niche. Nature 459, 262-265 (2009).

22. Huch, M. et al. In vitro expansion of single Lgr5+ liver stem cells induced by Wnt-driven regeneration. Nature 494, 247-250 (2013).

23. Carmon, K. S., Gong, X., Lin, Q., Thomas, A. \& Liu, Q. R-spondins function as ligands of the orphan receptors LGR4 and LGR5 to regulate Wnt/beta-catenin signaling. Proc. Natl Acad. Sci. USA 108, 11452-11457 (2011).

24. Glinka, A. et al. LGR4 and LGR5 are R-spondin receptors mediating Wnt/ beta-catenin and Wnt/PCP signalling. EMBO Rep. 12, 1055-1061 (2011).

25. Koo, B. K. et al. Tumour suppressor RNF43 is a stem-cell E3 ligase that induces endocytosis of Wnt receptors. Nature 488, 665-669 (2012).

26. Hao, H. X. et al. ZNRF3 promotes Wnt receptor turnover in an R-spondinsensitive manner. Nature 485, 195-200 (2012). 
27. Szenker-Ravi, E. et al. RSPO2 inhibition of RNF43 and ZNRF3 governs limb development independently of LGR4/5/6. Nature 557, 564-569 (2018).

28. Kim, K. A. et al. R-Spondin family members regulate the Wnt pathway by a common mechanism. Mol. Biol. Cell 19, 2588-2596 (2008).

29. Ohkawara, B., Glinka, A. \& Niehrs, C. Rspo3 binds syndecan 4 and induces Wnt/PCP signaling via clathrin-mediated endocytosis to promote morphogenesis. Dev. Cell 20, 303-314 (2011).

30. Lebensohn, A. M. \& Rohatgi, R. R-spondins can potentiate WNT signaling without LGRs. Elife 7, e33126 (2018)

31. Yan, K. S. et al. Non-equivalence of Wnt and R-spondin ligands during Lgr5 (+) intestinal stem-cell self-renewal. Nature 545, 238-242 (2017).

32. Klauzinska, M. et al. Rspo2/Int7 regulates invasiveness and tumorigenic properties of mammary epithelial cells. J. Cell Physiol. 227, 1960-1971 (2012).

33. Mulvaney, J. F. et al. Secreted factor R-Spondin 2 is involved in refinement of patterning of the mammalian cochlea. Dev. Dyn. 242, 179-188 (2013).

34. Janda, C. Y. et al. Surrogate Wnt agonists that phenocopy canonical Wnt and beta-catenin signalling. Nature 545, 234-237 (2017).

35. Harland, R. \& Gerhart, J. Formation and function of Spemann's organizer. Annu. Rev. Cell Dev. Biol. 13, 611-667 (1997).

36. De Robertis, E. M., Larrain, J., Oelgeschlager, M. \& Wessely, O. The establishment of Spemann's organizer and patterning of the vertebrate embryo. Nat. Rev. Genet. 1, 171-181 (2000).

37. Niehrs, C. Regionally specific induction by the Spemann-Mangold organizer. Nat. Rev. Genet. 5, 425-434 (2004).

38. Reversade, B., Kuroda, H., Lee, H., Mays, A. \& De Robertis, E. M. Depletion of Bmp2, Bmp4, Bmp7 and Spemann organizer signals induces massive brain formation in Xenopus embryos. Development 132, 3381-3392 (2005).

39. Kirsch, N. et al. Angiopoietin-like 4 Is a Wnt Signaling Antagonist that Promotes LRP6 Turnover. Dev. Cell 43, 71-82 e76 (2017).

40. Niehrs, C. \& Pollet, N. Synexpression groups in eukaryotes. Nature 402, 483-487 (1999).

41. Karaulanov, E., Knochel, W. \& Niehrs, C. Transcriptional regulation of BMP4 synexpression in transgenic Xenopus. EMBO J. 23, 844-856 (2004).

42. Paulsen, M., Legewie, S., Eils, R., Karaulanov, E. \& Niehrs, C. Negative feedback in the bone morphogenetic protein 4 (BMP4) synexpression group governs its dynamic signaling range and canalizes development. Proc. Natl Acad. Sci. USA 108, 10202-10207 (2011).

43. Chang, L. S., Kim, M., Glinka, A., Reinhard, C. \& Niehrs, C. The tumor suppressor PTPRK promotes ZNRF3 internalization and is required for Wnt inhibition in the Spemann organizer. Elife 9, e51248 (2020).

44. Onichtchouk, D. et al. Silencing of TGF-beta signalling by the pseudoreceptor BAMBI. Nature 401, 480-485 (1999).

45. Tsang, H. T. et al. The hereditary spastic paraplegia proteins NIPA1, spastin and spartin are inhibitors of mammalian BMP signalling. Hum. Mol. Genet. 18, 3805-3821 (2009).

46. Ide, H. et al. Cloning of human bone morphogenetic protein type IB receptor (BMPR-IB) and its expression in prostate cancer in comparison with other BMPRs. Oncogene 14, 1377-1382 (1997).

47. Fisher, M. C., Li, Y., Seghatoleslami, M. R., Dealy, C. N. \& Kosher, R. A. Heparan sulfate proteoglycans including syndecan-3 modulate BMP activity during limb cartilage differentiation. Matrix Biol. 25, 27-39 (2006).

48. Olivares, G. H. et al. Syndecan-1 regulates BMP signaling and dorso-ventral patterning of the ectoderm during early Xenopus development. Dev. Biol. 329, 338-349 (2009).

49. Reis, A. H. \& Sokol, S. Y. Rspo2 antagonizes FGF signaling during vertebrate mesoderm formation and patterning. Development 147, dev189324 (2020).

50. Schille, C., Heller, J. \& Schambony, A. Differential requirement of bone morphogenetic protein receptors Ia (ALK3) and Ib (ALK6) in early embryonic patterning and neural crest development. BMC Dev. Biol. 16, 1 (2016).

51. Leibovich, A., Steinbeisser, H. \& Fainsod, A. Expression of the ALK1 family of type I BMP/ADMP receptors during gastrula stages in Xenopus embryos. Int. J. Dev. Biol. 61, 465-470 (2017).

52. Nakayama, T. et al. Xenopus Smad8 acts downstream of BMP-4 to modulate its activity during vertebrate embryonic patterning. Development 125, 857-867 (1998).

53. Bachiller, D. et al. The organizer factors Chordin and Noggin are required for mouse forebrain development. Nature 403, 658-661 (2000).

54. Khokha, M. K., Yeh, J., Grammer, T. C. \& Harland, R. M. Depletion of three BMP antagonists from Spemann's organizer leads to a catastrophic loss of dorsal structures. Dev. Cell 8, 401-411 (2005).

55. Blitz, I. L., Cho, K. W. \& Chang, C. Twisted gastrulation loss-of-function analyses support its role as a BMP inhibitor during early Xenopus embryogenesis. Development 130, 4975-4988 (2003).

56. Dal-Pra, S., Furthauer, M., Van-Celst, J., Thisse, B. \& Thisse, C. Noggin1 and Follistatin-like2 function redundantly to Chordin to antagonize BMP activity. Dev. Biol. 298, 514-526 (2006).

57. Nam, J. S., Turcotte, T. J. \& Yoon, J. K. Dynamic expression of R-spondin family genes in mouse development. Gene Expr. Patterns 7, 306-312 (2007).
58. Bell, S. M. et al. R-spondin 2 is required for normal laryngeal-tracheal, lung and limb morphogenesis. Development 135, 1049-1058 (2008).

59. Nam, J. S. et al. Mouse R-spondin2 is required for apical ectodermal ridge maintenance in the hindlimb. Dev. Biol. 311, 124-135 (2007).

60. Fujii, M. et al. Roles of bone morphogenetic protein type I receptors and Smad proteins in osteoblast and chondroblast differentiation. Mol. Biol. Cell 10, 3801-3813 (1999).

61. Goto, K., Kamiya, Y., Imamura, T., Miyazono, K. \& Miyazawa, K. Selective inhibitory effects of Smad6 on bone morphogenetic protein type I receptors. J. Biol. Chem. 282, 20603-20611 (2007).

62. Gawantka, V., Delius, H., Hirschfeld, K., Blumenstock, C. \& Niehrs, C. Antagonizing the Spemann organizer: role of the homeobox gene Xvent-1. EMBO J. 14, 6268-6279 (1995).

63. Nakayama, T. et al. Cas9-based genome editing in Xenopus tropicalis. Methods Enzymol. 546, 355-375 (2014).

64. Berger, B. S., Acebron, S. P., Herbst, J., Koch, S. \& Niehrs, C. Parkinson's disease-associated receptor GPR37 is an ER chaperone for LRP6. EMBO Rep. 18, 712-725 (2017).

\section{Acknowledgements}

We thank F. Cong for providing the ZNRF3 constructs; D. Koinuma for providing the ALK constructs; C. Janda for providing the WNT surrogate construct; R. Thomas for H1581 cells. We acknowledge G. Roth and Aska Pharmaceuticals Tokyo for generously providing hCG. We thank NXR (RRID: SCR_013731), Xenbase (RRID: SCR_004337), and EXRC for Xenopus resources. We thank Fabio da Silva for critical reading of the manuscript. Expert technical support by the DKFZ core facility for light microscopy and the central animal laboratory of DKFZ is gratefully acknowledged. This work was funded by the Deutsche Forschungsgemeinschaft (DFG, German Research Foundation) SFB1324 - project number 331351713.

\section{Author contributions}

H.L. designed and conducted in vitro, human cell line and Xenopus experiments. C.S. conducted human cell line and Xenopus experiments. R.S. designed and carried out in vitro and human cell line experiments. A.G. generated materials for the study. H.L. generated illustrations for schematics and models used in the study. All authors analyzed and discussed the data. C.N. conceived and coordinated the study and wrote the paper with contribution from H.L.

\section{Funding}

Open Access funding enabled and organized by Projekt DEAL.

\section{Competing interests}

The authors declare no competing interests.

\section{Additional information}

Supplementary information is available for this paper at https://doi.org/10.1038/s41467 020-19373-w.

Correspondence and requests for materials should be addressed to C.N.

Peer review information Nature Communications thanks Bruno Reversade and other, anonymous, reviewers for their contributions to the peer review of this work. Peer review reports are available.

Reprints and permission information is available at http://www.nature.com/reprints

Publisher's note Springer Nature remains neutral with regard to jurisdictional claims in published maps and institutional affiliations.

Open Access This article is licensed under a Creative Commons Attribution 4.0 International License, which permits use, sharing, adaptation, distribution and reproduction in any medium or format, as long as you give appropriate credit to the original author(s) and the source, provide a link to the Creative Commons license, and indicate if changes were made. The images or other third party material in this article are included in the article's Creative Commons license, unless indicated otherwise in a credit line to the material. If material is not included in the article's Creative Commons license and your intended use is not permitted by statutory regulation or exceeds the permitted use, you will need to obtain permission directly from the copyright holder. To view a copy of this license, visit http://creativecommons.org/ licenses/by/4.0/

(c) The Author(s) 2020 